\title{
Stereo Disparity through Cost Aggregation with Guided Filter
}

\author{
Pauline Tan $^{1}$, Pascal Monasse ${ }^{2}$ \\ ${ }^{1}$ CMLA, ENS Cachan, France (pauline.tan@ens-cachan.fr) \\ ${ }^{2}$ Université Paris-Est, LIGM (UMR CNRS 8049), ENPC, F-77455 Marne-la-Vallée (monasse@imagine.enpc.fr) \\ Communicated by Andrés Almansa Demo edited by Pascal Monasse
}

\begin{abstract}
Estimating the depth, or equivalently the disparity, of a stereo scene is a challenging problem in computer vision. The method proposed by Rhemann et al. in 2011 is based on a filtering of the cost volume, which gives for each pixel and for each hypothesized disparity a cost derived from pixel-by-pixel comparison. The filtering is performed by the guided filter proposed by He et al. in 2010. It computes a weighted local average of the costs. The weights are such that similar pixels tend to have similar costs. Eventually, a winner-take-all strategy selects the disparity with the minimal cost for each pixel. Non-consistent labels according to left-right consistency are rejected; a densification step can then be launched to fill the disparity map. The method can be used to solve other labeling problems (optical flow, segmentation) but this article focuses on the stereo matching problem.
\end{abstract}

\section{Source Code}

A software written in $\mathrm{C}++$ is available on the IPOL web page of this article ${ }^{1}$, which is the code used in the online demo. This gives similar results to the original authors' Matlab implementation $^{2}$. The program needs several parameters (see Section 4 for more detailed explanations). By default they are tuned as suggested in the original article, but one can adapt them to get better results.

\section{Supplementary Material}

In the demo, an optional rectification step can be launched before running the algorithm. The source code for this preprocessing step (not reviewed) can be found at the IPOL web page of this article ${ }^{3}$.

Keywords: stereo-matching; cost volume; guided filter

\footnotetext{
${ }^{1}$ https://doi.org/10.5201/ipol.2014.78

${ }^{2}$ https://www.ims.tuwien.ac.at/publications/tuw-202088

${ }^{3}$ https://doi.org/10.5201/ipol.2014.78
} 


\section{Introduction}

Stereo matching algorithms aim at estimating the depth of a scene given two photographs taken from different points of view (Figure 1). Depth estimation is done by estimating the disparity of each pixel, namely its apparent displacement from the first (reference) image to the second (target) image. This disparity permits to recover the 3D position of the point that was photographed in this pixel, if the stereo setup has been previously calibrated.

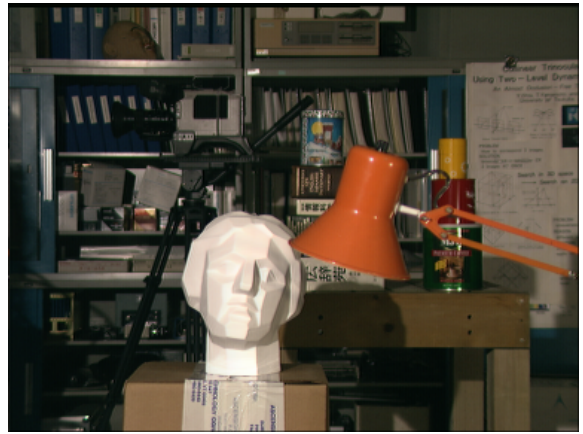

(a) Left (reference) image

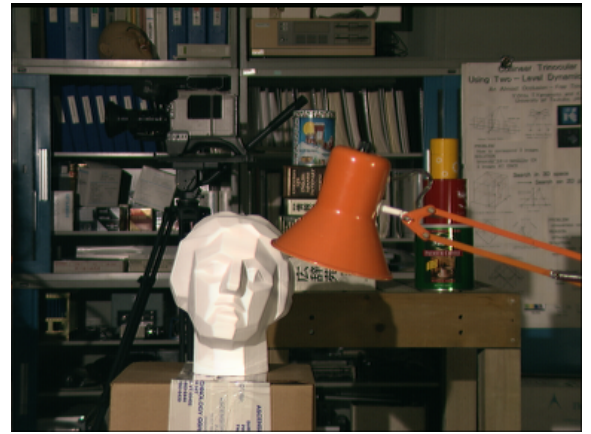

(b) Right (target) image

Figure 1: Tsukuba stereo pair

We assume that the left image is the reference image. Let $M$ be a $3 \mathrm{D}$-point. We denote by $O_{L}$ (resp. $O_{R}$ ) the principal point of the left (resp. right) camera, that is the orthogonal projection of the optical center on the image plane. The point $p$ (resp. $q$ ) denotes the image of $M$ in the left image (resp. in the right image). Then, the disparity of $M$ is given by $d(M)=\overrightarrow{O_{R} q}-\overrightarrow{O_{L} p}$. Assume the camera makes a so-called fronto-parallel move, meaning that the focal plane is the same for all positions of the camera and that its motion is parallel to the x-axis of the camera CCD array. This is the case for instance in the Middlebury benchmark dataset ${ }^{4}$. Then, the disparity is scalar and its absolute value is inversely proportional to the distance from the observer. More precisely, if the camera moves from the left to the right, any point has a negative disparity (see Figure 2). Hence, the lower the (signed) disparity, the lower the depth.

In more general cases, the situation is slightly different. After stereo-rectification, some information is lost, such as the position of the camera principal points. The coordinates of pixels in the images are then not given with respect to the optical center, but to an arbitrary point (denoted $O_{L}^{\prime}$ and $O_{R}^{\prime}$ ). Without any supplementary information, the disparity of a point $M$ is then given by $d^{\prime}(M)=\overrightarrow{O_{R}^{\prime} q}-\overrightarrow{O_{L}^{\prime} p}$. We see that we can go back to the previous case by writing $d^{\prime}(M)=\overrightarrow{O_{R}^{\prime} O_{R}}+\overrightarrow{O_{R} q}-\overrightarrow{O_{L} p}-\overrightarrow{O_{L}^{\prime} O_{L}}=d(M)+C$ where $C$ does not depend on the point $M$. Then, to recover the depth from the disparity of any point, a constant has to be added to the computed disparity, but lower disparity still means lower depth.

If the camera motion is right to left, the situation is inverted and lower disparity means higher depth. This is important for the occlusion filling process, which in a first step fills in the non informed pixels with a disparity corresponding to lower depth.

\section{Algorithm Description}

Let $I_{L}$ and $I_{R}$ be respectively the left and right color images of a stereo pair, of size $M \times N$. Then, $I_{L}^{r}, I_{L}^{g}$ and $I_{L}^{b}$ denote the color channels of the left image $\left(I_{R}^{r}, I_{R}^{g}\right.$ and $I_{R}^{b}$ for the right image). We

\footnotetext{
${ }^{4}$ http://vision.middlebury.edu/stereo/
} 

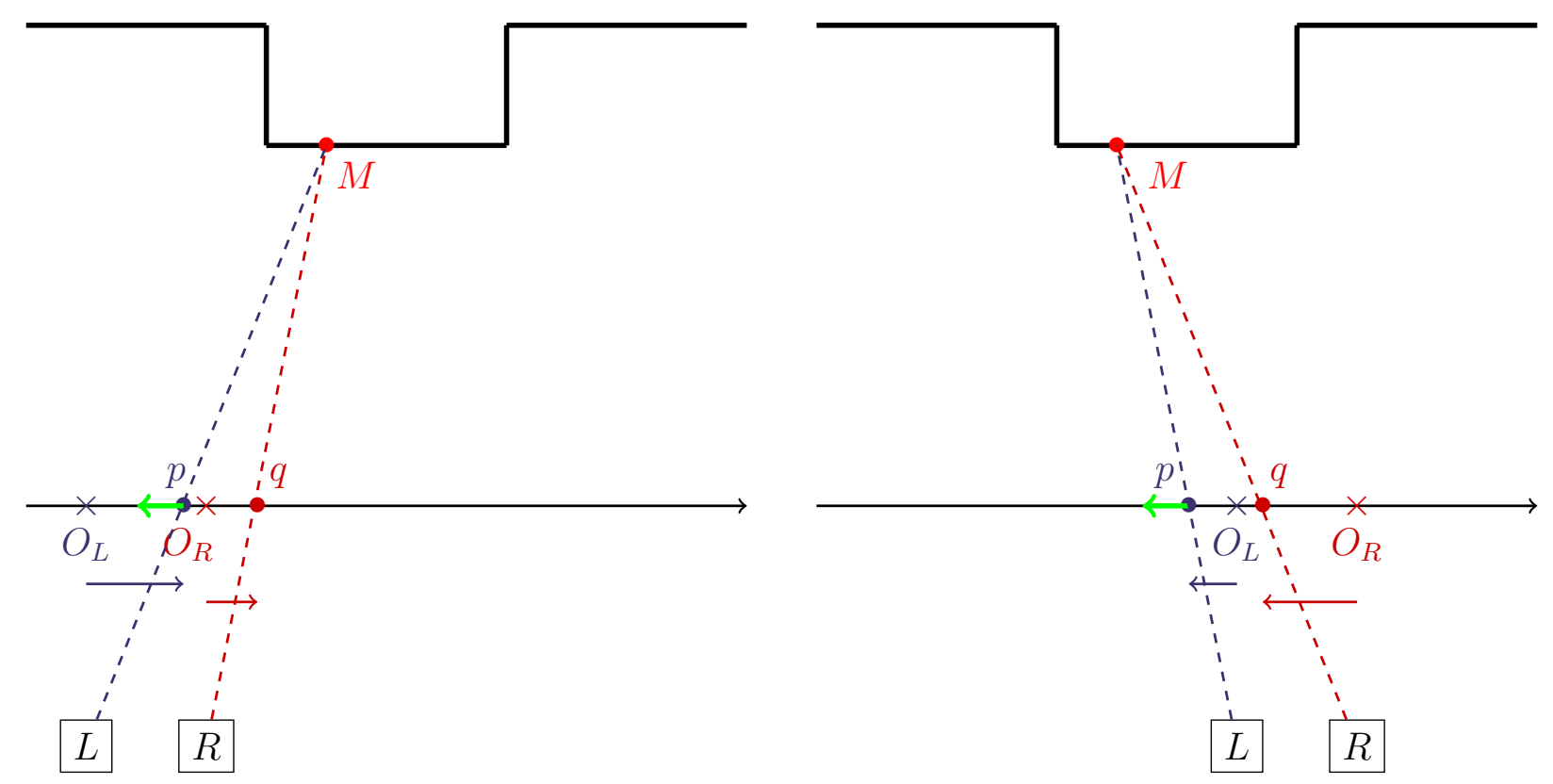

Figure 2: Disparity when the camera moves from the left to the right. The disparity of the pixel $M$ is given by subtracting the blue vector from the red vector. The disparity (in green) is then always negative.

proceed to the gray-level conversion of the images:

$$
\tilde{I}_{L}=0.299 \cdot I_{L}^{r}+0.587 \cdot I_{L}^{g}+0.0721 \cdot I_{L}^{b} \quad \text { and } \quad \tilde{I}_{R}=0.299 \cdot I_{R}^{r}+0.587 \cdot I_{R}^{g}+0.0721 \cdot I_{R}^{b} .
$$

We assume the disparity range $\left[d_{\min }, d_{\max }\right]$ to be known.

\subsection{Cost Volume}

Pixel-to-pixel comparison is done by involving the truncated absolute difference of the color and the $x$-derivative of the grayscale images. These two terms are balanced with a parameter $\alpha$.

1. Color comparison: for each pixel $i$ in the left image and each pixel $j$ in the right image, the color penalty is obtained by averaging the AD (Absolute Difference) on each color channel:

$$
N_{\text {color }}(i, j):=\frac{1}{3}\left(\left|I_{L}^{r}(i)-I_{R}^{r}(j)\right|+\left|I_{L}^{g}(i)-I_{R}^{g}(j)\right|+\left|I_{L}^{b}(i)-I_{R}^{b}(j)\right|\right)
$$

which is then thresholded:

$$
N_{\text {color }}^{\tau_{1}}(i, j):=\min \left(N_{\text {color }}(i, j), \tau_{1}\right)
$$

2. $x$-derivative comparison: the discrete derivative along the $x$-axis of the gray-level image $\tilde{I}_{L}$ $\left(\right.$ resp. $\left.\tilde{I}_{R}\right)$, denoted $\nabla_{x} \tilde{I}_{L}\left(\right.$ resp. $\left.\nabla_{x} \tilde{I}_{R}\right)$, is computed as follows ${ }^{5}$

$$
\forall i=\left(i_{x}, i_{y}\right), \quad \nabla_{x} \tilde{I}_{L}(i):=\frac{\tilde{I}_{L}\left(i_{x}+1, i_{y}\right)-\tilde{I}_{L}\left(i_{x}-1, i_{y}\right)}{2} .
$$

The AD is then computed

$$
N_{\text {gradient }}(i, j):=\left|\nabla_{x} \tilde{I}_{L}(i)-\nabla_{x} \tilde{I}_{R}(j)\right|
$$

\footnotetext{
${ }^{5}$ In (4), pixel intensities outside the image are considered to be the same as at the closest pixel inside the image.
} 
and thresholded

$$
N_{\text {gradient }}^{\tau_{2}}(i, j):=\min \left(N_{\text {gradient }}(i, j), \tau_{2}\right)
$$

3. Eventually, the color and gradient terms are balanced:

$$
N(i, j):=(1-\alpha) \cdot N_{\text {color }}^{\tau_{1}}(i, j)+\alpha \cdot N_{\text {gradient }}^{\tau_{2}}(i, j) .
$$

The cost volume $C$ is then defined on a sampling integer grid of the volume $[0, M-1] \times[0, N-1] \times$ $\left[d_{\min }, d_{\max }\right]$, such that, for any disparity value $d \in\left[d_{\min }, d_{\max }\right]$ :

$$
C(i, d):=N(i, i+d)
$$

This assumes that $i+d$ is in the domain of the right image (we note by a slight abuse of notation $i+d:=\left(i_{x}+d, i_{y}\right)$ if $\left.i=\left(i_{x}, i_{y}\right)\right)$. If it is not the case, we set $C(i, d):=(1-\alpha) \cdot \tau_{1}+\alpha \cdot \tau_{2}$ which is the maximal possible cost.

\section{2 $\quad$ Filtering with Guided Filter}

The pixel-to-pixel comparison alone is too sensitive to noise. So, each slice $p(i)=C(i, d)$ has to be filtered. The guided filter [4] is chosen since it is designed to preserve edges, by using the left image $I_{L}$ as guidance image.

Grayscale guidance image. Let $I$ be the guidance (grayscale) image and $q$ be the output of the filter. The key assumption of the guided filter is that it is a local linear model between $I$ and $q$. For each square window $\omega_{k}$ with radius $r_{\mathrm{GF}}$ centered at $k$ (by definition, a square with integer radius $r$ is a square with side length $2 \times r+1$ and $\left|\omega_{k}\right|$ denotes the number of pixels in $\omega_{k}$ ), a $k$-dependent filtered image $q_{k}$ is defined to be a linear transform of $I$ in $\omega_{k}$

$$
\exists a_{k}, b_{k}, \forall i \in \omega_{k}, \quad q_{k}(i)=a_{k} \cdot I(i)+b_{k} .
$$

If $k$ is too close to the image boundaries, then we only take into account the part of the window that remains in the domain of the image (see Figure 3).

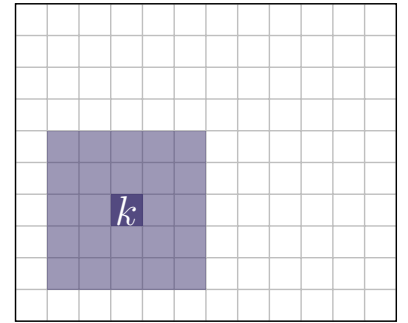

(a) Window of radius $r_{\mathrm{GF}}=2$ centered at $k=(3,6) .\left|\omega_{k}\right|=25$

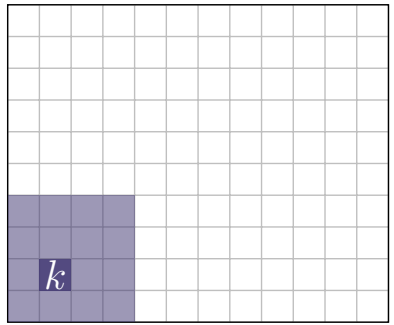

(b) Window of radius $r_{\mathrm{GF}}=2$ centered at $k=(1,8) .\left|\omega_{k}\right|=16$.

Figure 3: Examples of square windows.

In the window $\omega_{k}$, since $a_{k}$ and $b_{k}$ are constant, we can write

$$
\nabla q_{k}=a_{k} \nabla I .
$$


That is, strong edges in $I$ imply strong edges in $q_{k}$. Hence, the guided filter preserves the edges of the guidance image. To determine coefficients $a_{k}$ and $b_{k}$, the $\mathrm{L}^{2}$-norm between the filter input $p$ and the filter output $q_{k}$ is minimized. Hence, an energy-based formulation of this problem is:

$$
\arg \min _{a_{k}, b_{k}}\left\{E\left(a_{k}, b_{k}\right):=\sum_{i \in \omega_{k}}\left(a_{k} \cdot I(i)+b_{k}-p(i)\right)^{2}+\varepsilon\left|\omega_{k}\right| a_{k}^{2}\right\} .
$$

The first term is a data term and the second term is a regularization term preventing $a_{k}$ from being too large. The parameters $a_{k}$ and $b_{k}$ are then obtained by solving the Euler equation $(\nabla E=0)$ :

$$
\begin{aligned}
& a_{k}= \frac{\frac{1}{\left|\omega_{k}\right|} \sum_{i \in \omega_{k}} I(i) p(i)-\mu_{k} \bar{p}_{k}}{\sigma_{k}^{2}+\varepsilon} \\
& b_{k}=\bar{p}_{k}-a_{k} \mu_{k}
\end{aligned}
$$

where $\mu_{k}$ and $\sigma_{k}^{2}$ are the mean and variance of $I$ in the window $\omega_{k}$, and $\bar{p}_{k}$ is the mean of $p$ in $\omega_{k}$ :

$$
\mu_{k}:=\frac{1}{\left|\omega_{k}\right|} \sum_{i \in \omega_{k}} I(i), \quad \sigma_{k}^{2}:=\frac{1}{\left|\omega_{k}\right|} \sum_{i \in \omega_{k}} I(i)^{2}-\mu_{k}^{2} \quad \text { and } \quad \bar{p}_{k}:=\frac{1}{\left|\omega_{k}\right|} \sum_{i \in \omega_{k}} p(i) .
$$

The coefficients computed above depend on the window $\omega_{k}$. However, any pixel $i$ is involved in several windows $\omega_{k}$, which leads to many values of coefficients $a_{k}$ and $b_{k}$. A simple way to solve this problem is to take the average of all these values:

$$
q(i):=\frac{1}{\left|\omega_{i}\right|} \sum_{k \mid i \in \omega_{k}} q_{k}(i)=\frac{1}{\left|\omega_{i}\right|} \sum_{k \mid i \in \omega_{k}}\left(a_{k} \cdot I(i)+b_{k}\right)=\bar{a}_{i} \cdot I(i)+\bar{b}_{i}
$$

with $\bar{a}_{i}\left(\right.$ resp. $\left.\bar{b}_{i}\right)$ the mean of $a_{k}$ (resp. $b_{k}$ ) in the window $\omega_{i}$, namely

$$
\bar{a}_{i}:=\frac{1}{\left|\omega_{i}\right|} \sum_{k \mid i \in \omega_{k}} a_{k} \quad \text { and } \quad \bar{b}_{i}:=\frac{1}{\left|\omega_{i}\right|} \sum_{k \mid i \in \omega_{k}} b_{k} .
$$

Notice that $i \in \omega_{k} \Leftrightarrow k \in \omega_{i}$, which explains the normalization factor $1 /\left|\omega_{i}\right|$.

The filter output $q$ is then computed following four steps:

1. Computing the images $\mu_{k}, \bar{p}_{k}, \frac{1}{\left|\omega_{k}\right|} \sum_{i \in \omega_{k}} I(i) p(i)$, and $\sigma_{k}^{2}$;

2. Computing the images $a_{k}$ and $b_{k}$ with expressions (12) and (13);

3. Computing the images $\bar{a}_{i}$ and $\bar{b}_{i}$ by (16);

4. Computing $q$ thanks to (15).

Note that the first three steps use a box filter (mean in a square window). An efficient implementation of the box filter, independent of the radius of the square window, is shown in the next section. The above filter may be better understood when reformulated as a variable kernel convolution $W^{\mathrm{GF}}$ :

$$
q(i)=\sum_{j} W_{i j}^{\mathrm{GF}} \cdot p(j) \quad \text { with } \quad W_{i j}^{\mathrm{GF}}:=\sum_{k \in \omega_{i} \cap \omega_{j}} \frac{1}{\left|\omega_{i}\right| \cdot\left|\omega_{k}\right|}\left(1+\frac{\left(I(j)-\mu_{k}\right)\left(I(i)-\mu_{k}\right)}{\sigma_{k}^{2}+\varepsilon}\right) .
$$

It is easily observed by this formula that pixels $j$ in the neighborhood of $i$ having similar color have a stronger weight in the resulting value of $q(i)$. 
Color guidance images. For color guidance images $\left(I=\left(I^{r}, I^{g}, I^{b}\right)^{T}\right)$, the linear model can be written as

$$
q_{k}(i)=a_{k}^{T} I(i)+b_{k}
$$

The vectors $a_{k}$ and $b_{k}$ are then given by

$$
a_{k}=\left(\Sigma_{k}+\varepsilon \mathrm{I}_{3}\right)^{-1}\left(\begin{array}{c}
\frac{1}{\left|\omega_{k}\right|} \sum_{i \in \omega_{k}} I^{r}(i) p(i)-\mu_{k}^{r} \bar{p}_{k} \\
\frac{1}{\left|\omega_{k}\right|} \sum_{i \in \omega_{k}} I^{g}(i) p(i)-\mu_{k}^{g} \bar{p}_{k} \\
\frac{1}{\left|\omega_{k}\right|} \sum_{i \in \omega_{k}} I^{b}(i) p(i)-\mu_{k}^{b} \bar{p}_{k}
\end{array}\right) \quad \text { and } \quad b_{k}=\bar{p}_{k}-a_{k}^{T}\left(\begin{array}{c}
\mu_{k}^{r} \\
\mu_{k}^{g} \\
\mu_{k}^{b}
\end{array}\right)
$$

with $\mu_{k}^{r}, \mu_{k}^{g}$, and $\mu_{k}^{b}$ the mean images of each color channel of the guidance image $I$ :

$$
\mu_{k}^{[1]}:=\frac{1}{\left|\omega_{k}\right|} \sum_{i \in \omega_{k}} I^{[1]}(i) \quad \text { for }[1] \in\{r, g, b\}
$$

$\Sigma_{k}$ is the covariance matrix of the guidance image $I$ in the window $\omega_{k}$,

$$
\Sigma_{k}:=\left(\begin{array}{ccc}
\sigma_{k}^{r r} & \sigma_{k}^{r g} & \sigma_{k}^{r b} \\
\sigma_{k}^{r g} & \sigma_{k}^{g g} & \sigma_{k}^{g b} \\
\sigma_{k}^{r b} & \sigma_{k}^{g b} & \sigma_{k}^{b b}
\end{array}\right) \text { where } \sigma_{k}^{[1][2]}:=\frac{1}{\left|\omega_{k}\right|} \sum_{i \in \omega_{k}} I^{[1]}(i) I^{[2]}(i)-\mu_{k}^{[1]} \mu_{k}^{[2]} \text { for }[1],[2] \in\{r, g, b\}
$$

and $\mathrm{I}_{3}$ is the identity matrix of size $3 \times 3$. In this case, the kernel weights are given by

$$
W_{i j}^{\mathrm{GF}}=\frac{1}{\left|\omega_{i}\right| \cdot\left|\omega_{j}\right|} \sum_{k \in \omega_{i} \cap \omega_{j}}\left(1+\left(I(i)-\mu_{k}\right)^{T}\left(\Sigma_{k}+\varepsilon \mathrm{I}_{3}\right)^{-1}\left(I(j)-\mu_{k}\right)\right) .
$$

In conclusion, each slice of the cost volume $C(\cdot, d)$ is filtered with the guidance image $I_{L}$ :

$$
C^{\text {filt }}(i, d):=\sum_{j \in \mathcal{L}} \frac{1}{\left|\omega_{i}\right| \cdot\left|\omega_{j}\right|} \sum_{k \in \omega_{i} \cap \omega_{j}}\left(1+\left(I_{L}(i)-\mu_{k}\right)^{T}\left(\Sigma_{k}+\varepsilon \mathrm{I}_{3}\right)^{-1}\left(I_{L}(j)-\mu_{k}\right)\right) \cdot C(j, d) .
$$

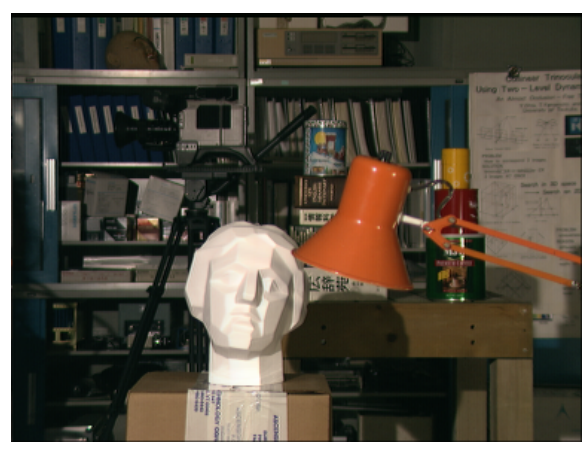

(a) Guidance image

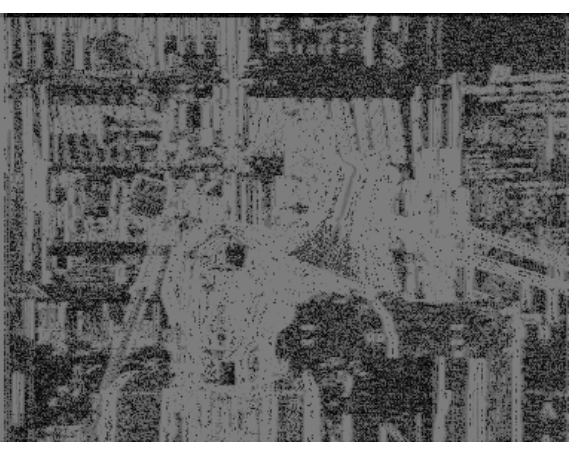

(b) Slice $d=-4$ of the cost volume

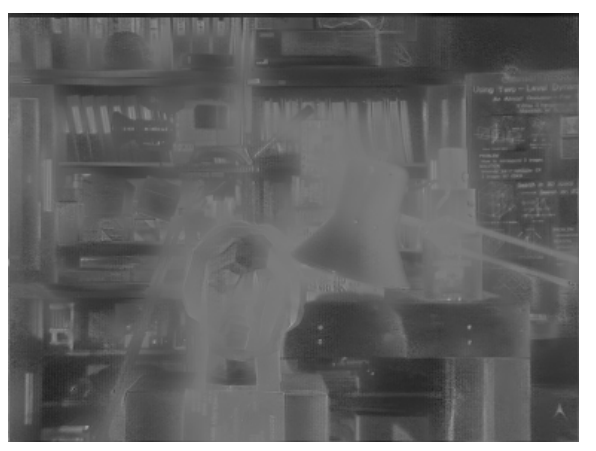

(c) Filtering by guided filter

Figure 4: Filtering by guided filter of a slice of the cost volume for the Tsukuba pair $\left(\tau_{1}=7, \tau_{2}=2\right.$, $\alpha=0.9, \varepsilon=10^{-4}, r_{\mathrm{GF}}=9$ ).

The generalization to multi-channel images as guidance, as for example hyper-spectral satellite imagery, would follow the same process with matrices of different size. 


\subsection{Implementation of the Box Filter [3]}

In this section, an implementation of the box filter proposed by Crow [2] is described. The simplest way to compute the mean in a square window of an image $I$ is to sum the value of each pixel of the window, and then divide by the window size. Thus, for a window size of $\left|\omega_{k}\right|,\left|\omega_{k}\right|-1$ sums and one division are needed. Hence, the complexity of the process is linear with the radius of the window.

Another way to do it would be to first compute an integral image $S$ of summed areas, of the same size as $I$ :

$$
\forall i=\left(i_{x}, i_{y}\right), \quad S(i)=\sum_{k_{x}=0}^{i_{x}} \sum_{k_{y}=0}^{i_{y}} I\left(k_{x}, k_{y}\right) .
$$

Then a sum in any rectangle $\left[i_{x}, j_{x}\right] \times\left[i_{y}, j_{y}\right]$ is given by (see Figure 5 )

$$
S\left(j_{x}, j_{y}\right)-S\left(i_{x}-1, j_{y}\right)-S\left(j_{x}, i_{y}-1\right)+S\left(i_{x}-1, i_{y}-1\right)
$$

an eventual negative index removing the involved terms of $S$ in the formula. Hence the computation of the mean only needs one sum, two subtractions and one division; it no longer depends on the radius of the window.

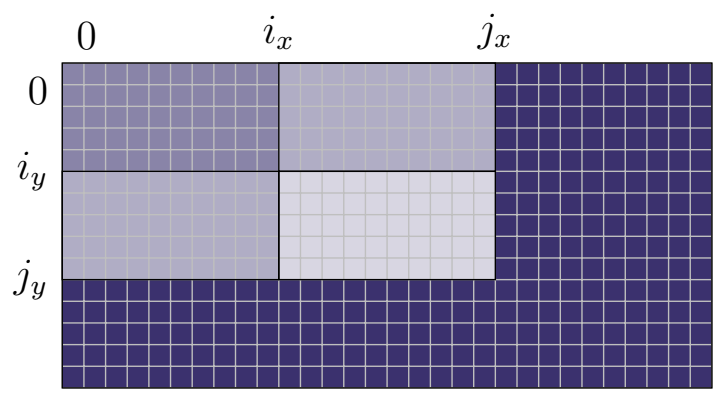

Figure 5: Calculation of a summed area from the integral image.

\subsection{Disparity Selection}

Once the cost volume is filtered, the disparity of any pixel $i$ is the disparity that minimizes the cost associated to $i$ in the cost volume ("Winner-take-all" strategy):

$$
d(i)=\arg \min _{d^{\prime} \in\left[d_{\min }, d_{\max }\right]}\left\{C^{\mathrm{filt}}\left(i, d^{\prime}\right)\right\} .
$$

\subsection{Left-Right Consistency}

The left-right consistency checks whether the disparity computed from the left image $I_{L}$ coincides with the disparity computed from the right image $I_{R}$. At pixel precision, a disparity is rejected when

$$
d(i) \neq-d^{\prime}(i+d(i))
$$

where $d^{\prime}$ denotes the disparity map computed from right to left image. This mask is expected to reject all occluded pixels and some flat region pixels of the scene. Thus rejected pixels are called "occluded pixels" in what follows. To handle subpixel disparities as well, this can be reformulated in the more general constraint:

$$
\left|d(i)+d^{\prime}(i+d(i))\right| \geq d_{L R}
$$

with $d_{L R}=1$. Equation $(27)$ is $(28)$ when $d_{L R}=0$. 


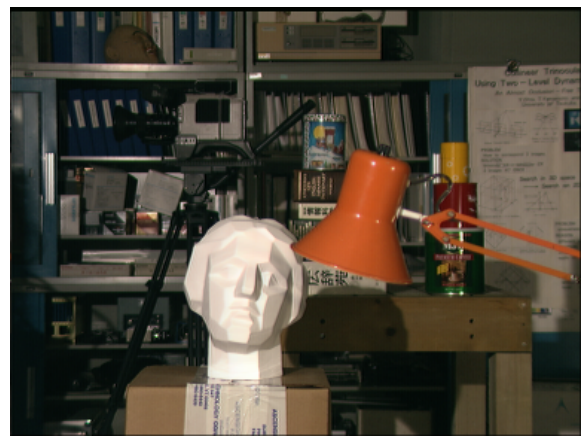

(a) Left image

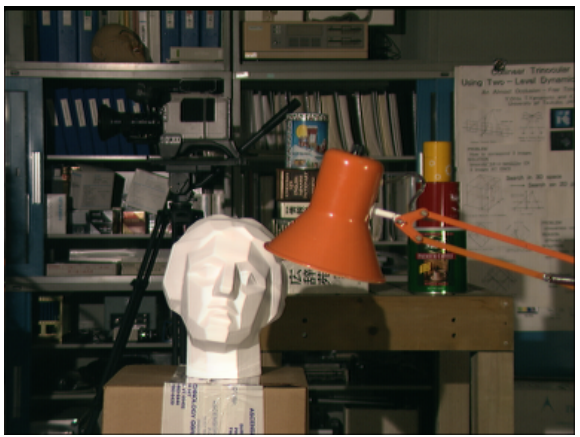

(b) Right image

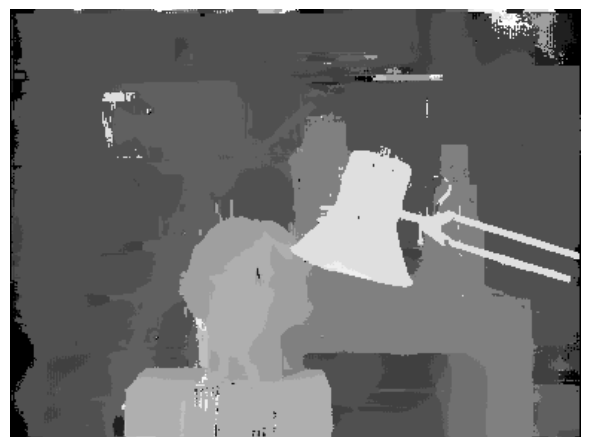

(c) Disparity map

Figure 6: Result of the winner-take-all algorithm on the Tsukuba pair.

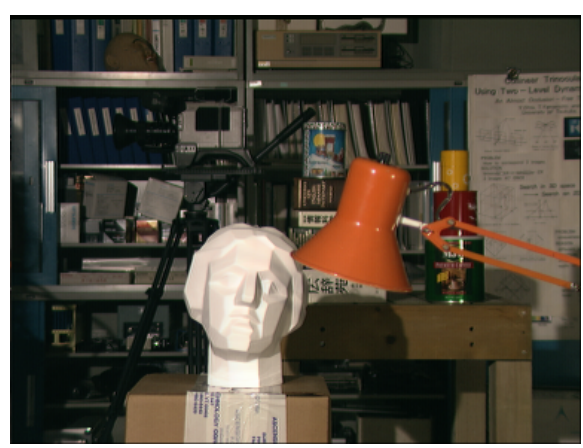

(a) Left image

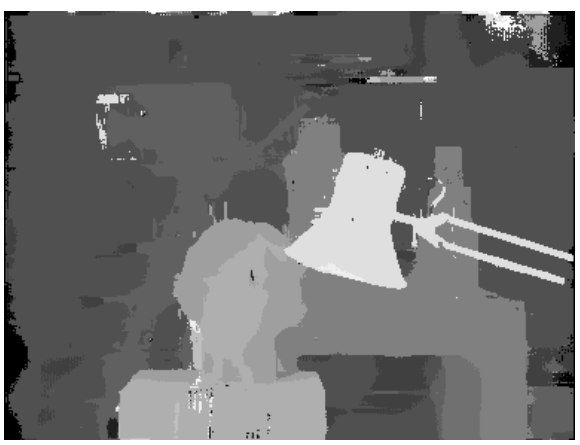

(b) Disparity map

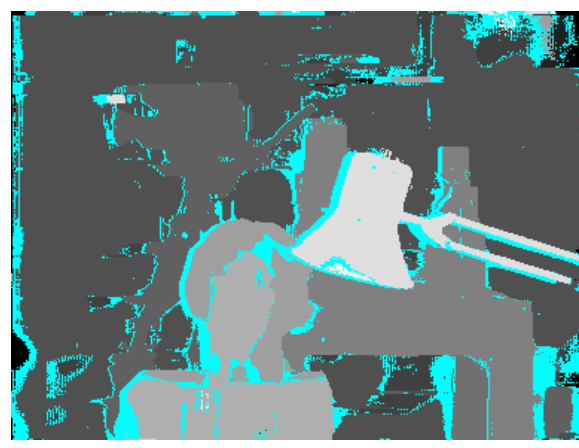

(c) Left-right consistency rejections

Figure 7: Result of the left-right consistency check on the Tsukuba pair. Most of the occluded pixels are detected by the left-right consistency mask. So are some pixels on constant disparity regions.

\subsection{Densification Process}

This step aims at filling the disparity map after the rejection test. Only occluded pixels are affected by this process, non-occluded ones keep their original disparity. It is done in two stages: the basic filling stage and the post-processing stage.

In the first stage a disparity value is given to the pixels rejected by the previous step. Since the mask mainly rejects occluded pixels, it often involves points of the scene that are hidden by other objects. In such cases, the rejected pixels are mainly in a region of discontinuity of the scene, where a closer object is in front of a farther one. Hence a strategy would be to adopt the disparity of the farther object, by exploring the closest nonoccluded pixels (on the same line). We recall that the farther object is given by the higher disparity. Hence, this leads to the following filling-in formula:

$$
\forall i=\left(i_{x}, i_{y}\right), d_{\text {fill }}(i):=\max \left\{\begin{array}{l}
d\left(i_{x}+\min _{\substack{j_{x} \leq 0 \\
\left(i_{x}+j_{x}, i_{y}\right) \text { nonoccluded }}}\left\{j_{x}\right\}, i_{y}\right) \\
d\left(i_{x}+\min _{\substack{j_{x} \geq 0 \\
\left(i_{x}+j_{x}, i_{y}\right) \text { nonoccluded }}}\left\{j_{x}\right\}, i_{y}\right)
\end{array}\right\} .
$$

Notice that if $i$ is nonoccluded, $d_{\text {fill }}(i)=d(i)$.

Simple filling can generate streak-line artifacts. Hence an edge-preserving filter is used to remove them, by smoothing the disparity map. The left image is first filtered by the median filter (applied on $3 \times 3$-windows): for $[1] \in\{r, g, b\}$,

$$
I_{L, \text { filt }}^{[1]}(i)=\operatorname{median}\left\{I_{L}^{[1]}(i+j) \mid j=\left(j_{x}, j_{y}\right): j_{x}, j_{y} \in\{-1,0,+1\}\right\} .
$$




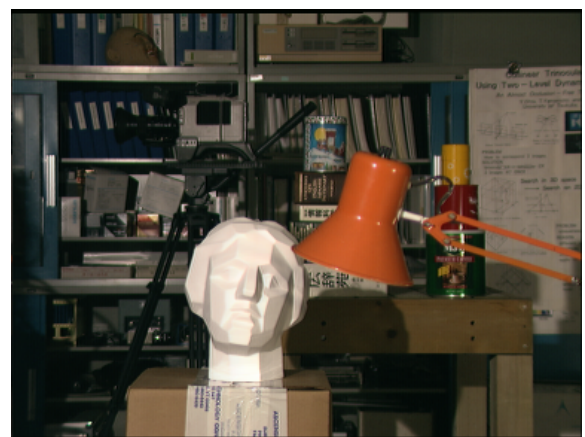

(a) Left image

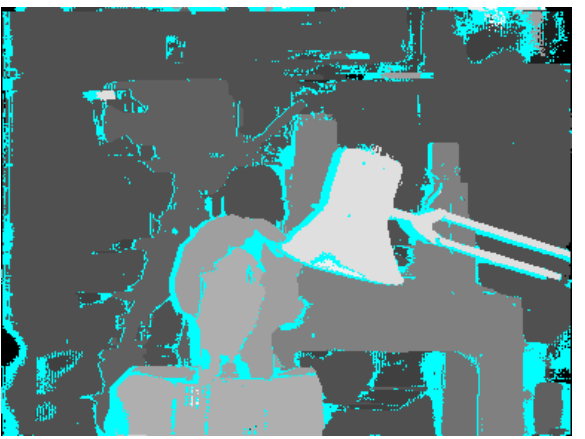

(b) Left-right consistency rejections

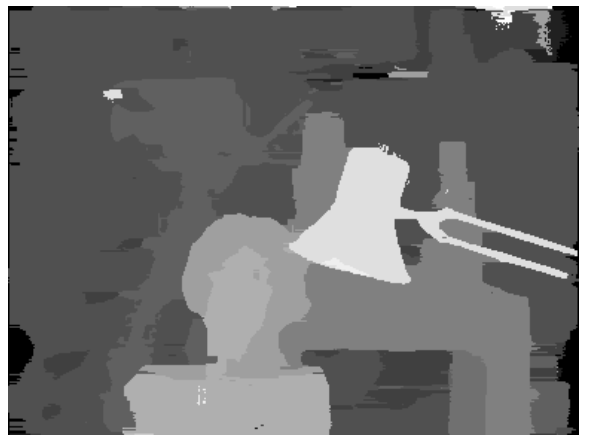

(c) Simple filling

Figure 8: Result of the filling on the Tsukuba pair. Streak-line artifacts appear.

Let $i$ be an occluded pixel. Then for each $j$ in the square window $\omega_{i}^{\prime}$ of radius $r_{\mathrm{WmF}}$ centered on $i$, the bilateral filter weight is computed by

$$
W_{i j}^{\mathrm{BF}}:=\exp \left(-\frac{\|i-j\|^{2}}{\sigma_{\mathrm{s}}^{2}}\right) \cdot \exp \left(-\frac{\left\|I_{L, \mathrm{filt}}(i)-I_{L, \mathrm{filt}}(j)\right\|_{c}^{2}}{\sigma_{\mathrm{c}}^{2}}\right),
$$

where $\|\cdot\|$ denotes the Euclidean distance and $\|\cdot\|_{c}$ the Euclidean distance in RGB color space:

$$
\left\|I_{L, \text { filt }}(i)-I_{L, \text { filt }}(j)\right\|_{c}^{2}=\left|I_{L, \text { filt }}^{r}(i)-I_{L, \text { filt }}^{r}(j)\right|^{2}+\left|I_{L, \text { filt }}^{g}(i)-I_{L, \text { filt }}^{g}(j)\right|^{2}+\left|I_{L, \text { filt }}^{b}(i)-I_{L, \text { filt }}^{b}(j)\right|^{2} .
$$

This filter gives higher weight to pixels spatially close and of similar color, which are likely to belong to the same object. A disparity cumulative histogram $h$ is then computed, where each value is weighted by the weight (31):

$$
\forall d \in\left[d_{\min }, d_{\max }\right], \quad h(d)=\sum_{j \in \omega_{i}^{\prime} \mid d(j) \leq d} W_{i j}^{\mathrm{BF}} .
$$

The final disparity of $i$ is then set to be the median value of $h$ :

$$
d_{\text {final }}(i):=\min \left\{d \mid h(d) \geq \frac{1}{2} h\left(d_{\max }\right)\right\} .
$$

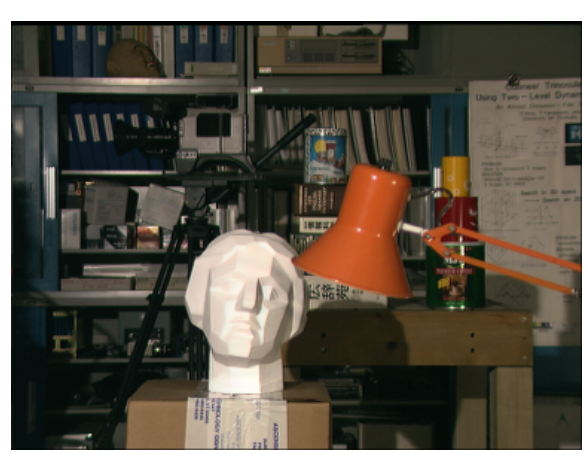

(a) Left image

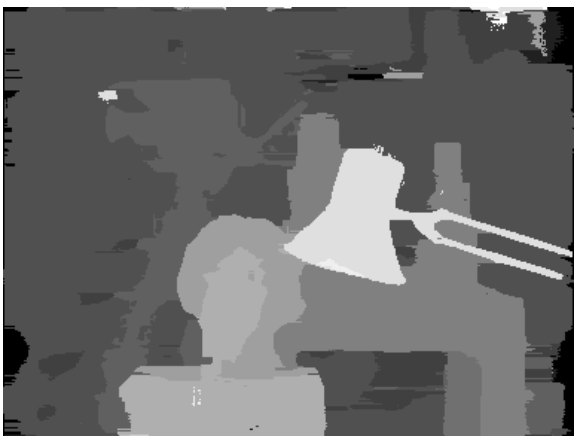

(b) Simple filling

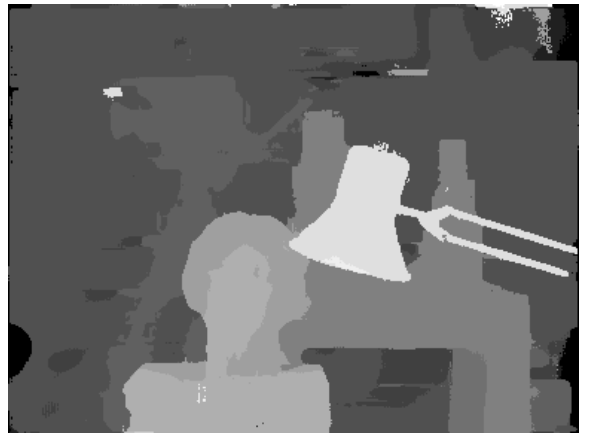

(c) Filtering the former by weighted median filter

Figure 9: Result of the post-processing on the Tsukuba pair. The disparity map is smoothed. 


\section{Algorithm Summary and Implementation}

A class Image defined in file image. $\mathrm{h}$ is used throughout. It is a container for an array of pixels that is reference counted: a copy of the image does not reallocate memory, it just adds a reference to the same array in memory (a coding pattern named shallow copy). The pixel by pixel operators of addition, subtraction and multiplication, used in the cost-volume filtering, are implemented as overloaded operators, easing readability of the code. Some simple image filters are implemented in file filters.cpp. Among them, boxFilter performs local averages using summed area tables. The method gradX computes the $x$-derivative of the image.

Algorithm 1 gives the pseudocode of the cost-volume filtering described above. The code is in file cost_volume.cpp. Notice that we do not need to store in memory the full cost-volume, only the slice at current disparity $d$ needs to be stored. The cost image $p$ is computed in function compute_cost. The filtering itself is in function filter_cost_volume. Our code implements the algorithm for color images even for actually grayscale images, which yields the same result, though at higher computation cost.

The consistency check is described in Algorithm 2. The disparity $d^{\prime}$ from $I_{R}$ to $I_{L}$ is computed, using range $\left[-d_{\max },-d_{\min }\right]$. The consistency between $d$ and $d^{\prime}$ is checked in function detect_occlusion of file occlusion.cpp. The threshold $d_{L R}$ ( 0 by default) is used.

The filling process is described in Algorithm 3. The simple filling is performed by using methods fillMaxX or fillMinX of class Image, depending on the camera motion direction (user input parameter). The filling based on bilateral weights (function fill_occlusion of file occlusion.cpp) is parallelized using openMP. Two functions in file filters.cpp are used: weighted_histo computes the local histogram of disparities weighted by bilateral weights, while median_histo computes the median of the histogram.

\section{Method Parameters}

Several parameters should be tuned to use this method. Table 1 recalls all of them. Default values of these parameters suggested in [8] are also given. Default values of $\tau_{1}, \tau_{2}, \varepsilon$ and $\sigma_{\mathrm{c}}$ are adapted to 8 -bit images with intensity range in $[0,255]$ (most frequent case), but should be changed for higher bit depths.

Results obtained with different values of the parameters are then shown in order to see their influence. The experiments were done on the Tsukuba pair. Unless explicitly stated otherwise, only one parameter is changed at a time. The other parameters are tuned by default. Errors are also shown (absolute disparity error $>0.5$ ) using a mask generated by Middlebury based on ground truth

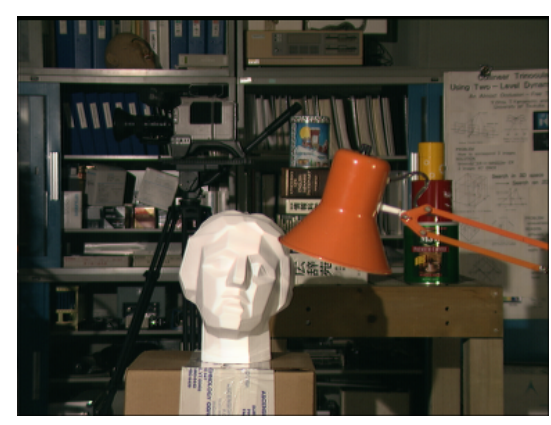

(a) Left image

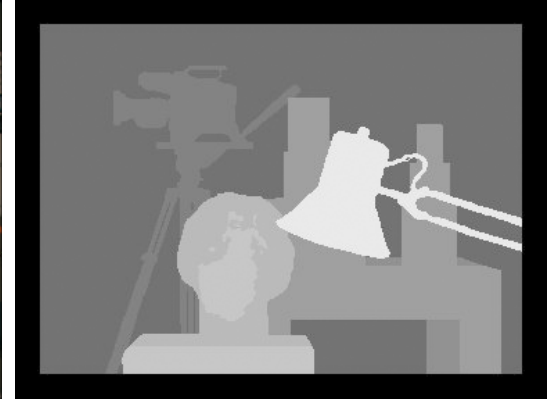

(b) Ground truth

Figure 10: Ground truth used to evaluate the results. 

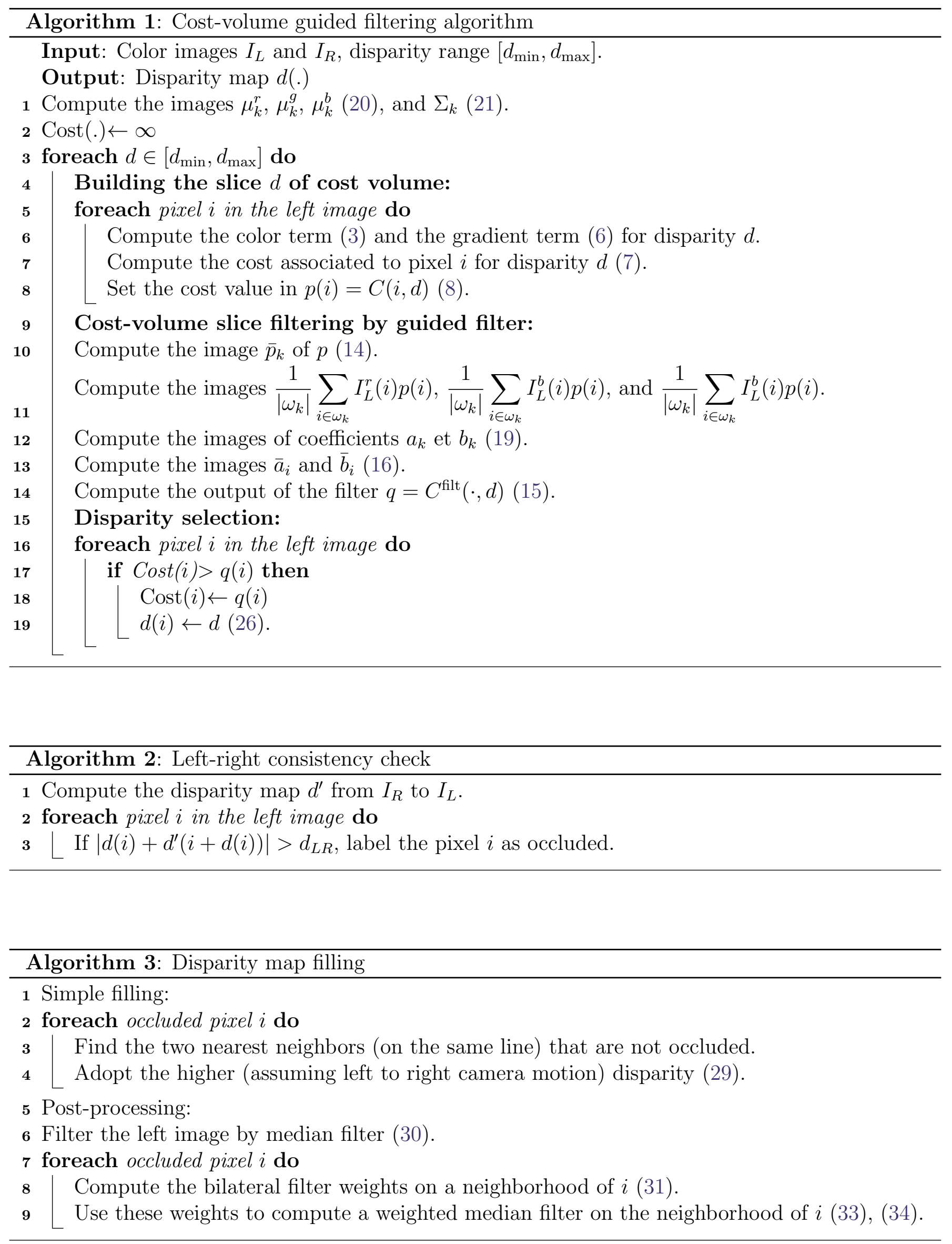


\begin{tabular}{|c|c|c|}
\hline Parameter & Suggested value & Description \\
\hline$r_{\mathrm{GF}}$ & 9 & Guided filter radius. \\
\hline$\alpha$ & 0.9 & $\begin{array}{l}\text { Parameter in }[0,1] \text { that balances the color and the } \\
\text { gradient contribution. The bigger this parameter, } \\
\text { the more important the contribution of the gradi- } \\
\text { ent term. }\end{array}$ \\
\hline$\tau_{1}$ & 7 & Threshold used for the color penalty AD distance. \\
\hline$\tau_{2}$ & 2 & $\begin{array}{l}\text { Threshold used for the gradient penalty AD dis- } \\
\text { tance. }\end{array}$ \\
\hline$\varepsilon$ & $255^{2} \cdot 10^{-4}$ & Guided filter regularization parameter. \\
\hline$d_{L R}$ & 0 & Left-right disparity difference tolerance \\
\hline$r_{\mathrm{WMF}}$ & 9 & Weighted median filter radius. \\
\hline$\sigma_{\mathrm{s}}$ & 9 & Weight of the spatial term in the bilateral filter. \\
\hline$\sigma_{\mathrm{c}}$ & $255 \cdot 10^{-1}$ & Weight of the color term in the bilateral filter. \\
\hline
\end{tabular}

Table 1: Method parameters.

measurements (Figure 10). Note that this mask does not take into account the image borders. These results were also submitted to the Middlebury site in order to automatically get the percentage of bad pixels (with a wrong disparity). In the following figures (Figure 11 to Figure 18), the first row shows the disparity map obtained by our implementation. The second row shows the disparity map with the mask (in pink) generated by Middlebury.

We first tested the influence of the parameters of the computation of the cost volume and its filtering by the guided filter. Figure 11 shows the results after the whole process (including filling and post-processing) when the size of the filter $r_{\mathrm{GF}}$ is tuned. A larger filter size gives smoother results often visually more satisfying. However, the disparity map can be too smooth and errors generated on the boundaries of some objects. Indeed, when we look at the percentage of bad pixels on the boundaries of objects, we get $19.7 \%$ bad pixels for $r_{\mathrm{GF}}=19$ while there are only $16.4 \%$ bad pixels for the default parameters. Nevertheless, the results remain globally better with a large filter size. Next, the influence of the parameter $\alpha$ was studied (see Figure 12). The larger this parameter, the heavier the gradient term weight. Results are better for large values of $\alpha$. In other terms, the gradient comparison seems much more reliable than the color comparison. This is due to the fact that the scene is textured. Note that the disparity estimation is better on the lamp when $\alpha$ is small, because the lamp has no texture.

Figure 13 shows the error percentages for four of the Middlebury images with respect to $r_{\mathrm{GF}}$ and $\alpha$, the two main parameters and the ones that can be tuned in the online demo. This shows that the default parameters are well adapted to get optimal results in the Middlebury benchmark.

The other parameters needed for the computation of the cost volume are the thresholds $\tau_{1}$ and $\tau_{2}$. By default, they are chosen very low, which limits the influence of a large difference (of color or gradient). Figure 14 shows that the results are more accurate when these thresholds are larger. The results degrade when the thresholds are too large, though.

The last parameter involved in the filtering of the cost volume is the regularization parameter $\varepsilon$. The larger this parameter, the smoother the disparity map. Figure 15 shows that even though the number of bad pixels is lower for larger values of $\varepsilon$, the results are not visually satisfactory since the boundaries of many objects seem loose while $\varepsilon$ grows.

The influence of the parameters involved in the filling process was also tested. We started with the size of the weighted median filter. Figure 16 shows that this parameter is not a key parameter, since the results do not significantly change when we tune it. The disparity estimation is slightly enhanced when the filter size grows. However, the larger the filter, the slower the computation. 


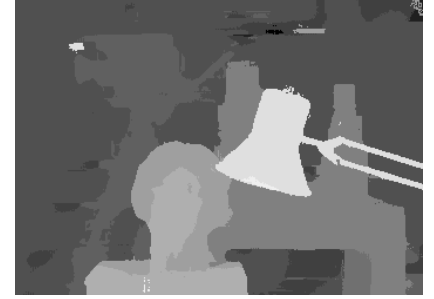

(a) $r_{\mathrm{GF}}=9$

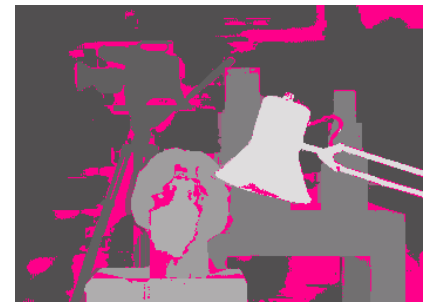

(e) $r_{\mathrm{GF}}=9$

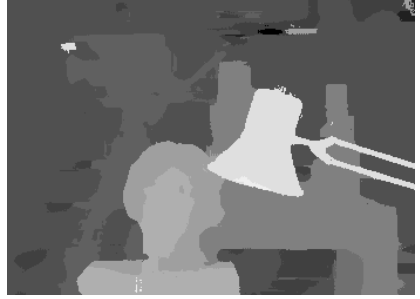

(b) $r_{\mathrm{GF}}=4$

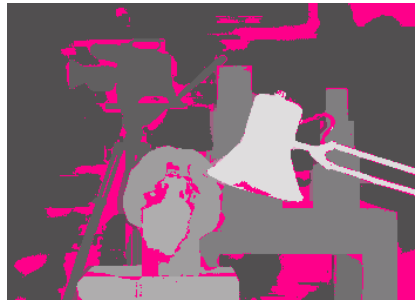

(f) $r_{\mathrm{GF}}=4$

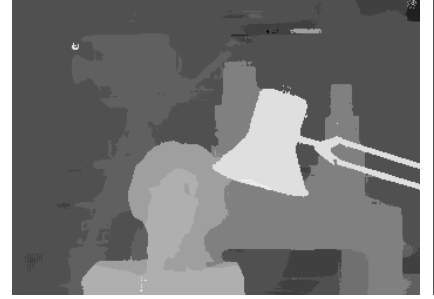

(c) $r_{\mathrm{GF}}=11$

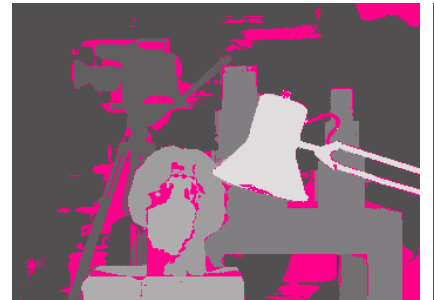

(g) $r_{\mathrm{GF}}=11$

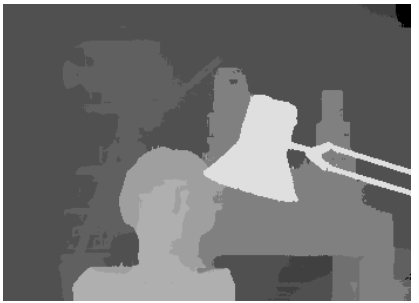

(d) $r_{\mathrm{GF}}=19$

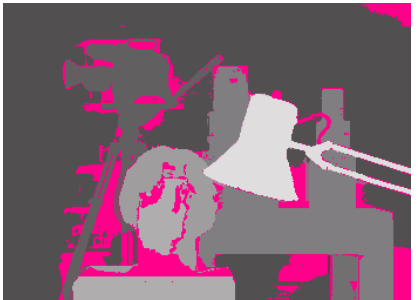

(h) $r_{\mathrm{GF}}=19$

Figure 11: Influence of the guided filter size. (a)+(e) Default parameters $\left(r_{\mathrm{GF}}=9\right) .12 .9 \%$ are bad pixels. (b) + (f) $r_{\mathrm{GF}}=4$, bad pixels: $15.9 \%$. (c) + (g) $r_{\mathrm{GF}}=11$, bad pixels: $11.8 \%$. (d) + (h) $r_{\mathrm{GF}}=19$, bad pixels: $11.7 \%$. When the filter size grows, the disparity map is cleaner (wrong disparities are removed, e.g. in the background), but errors appear on the object boundaries (e.g. on the space between the lamp and the table), due to an oversmooth map.

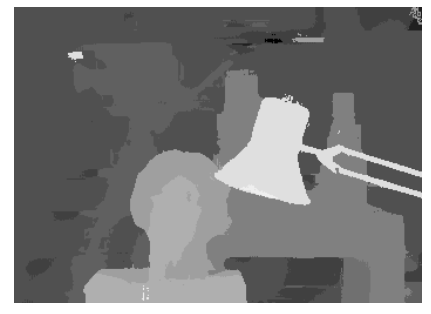

(a) $\alpha=0.9$

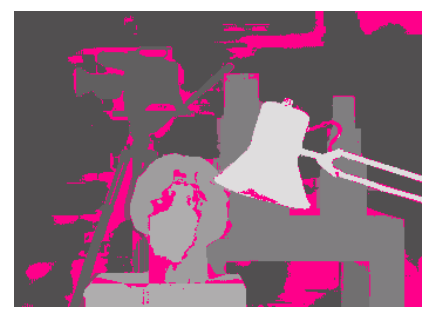

(e) $\alpha=0.9$

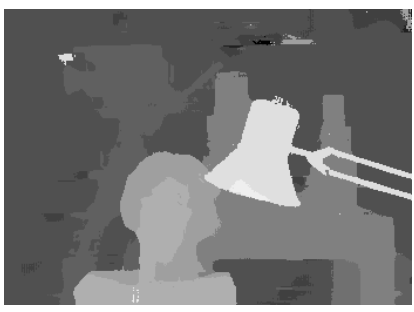

(b) $\alpha=0.95$

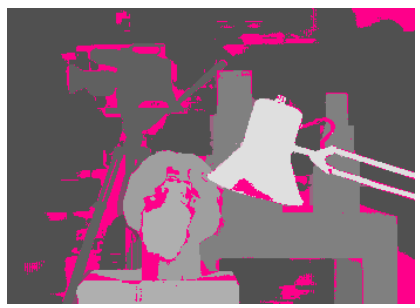

(f) $\alpha=0.95$

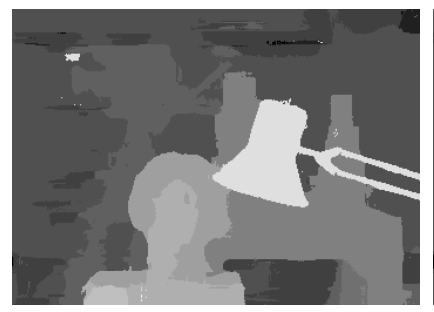

(c) $\alpha=0.5$

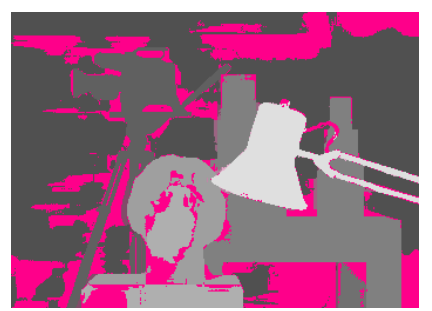

(g) $\alpha=0.5$

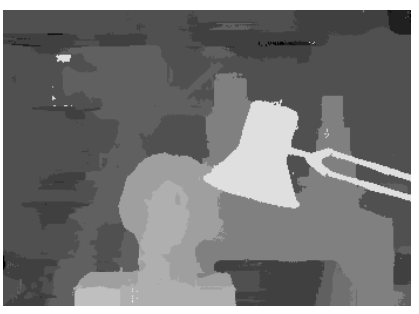

(d) $\alpha=0.1$

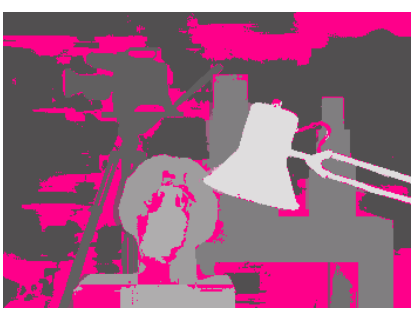

(h) $\alpha=0.1$

Figure 12: Influence of the parameter $\alpha$. (a)+(e) Default parameters $(\alpha=0.9)$, bad pixels: $12.9 \%$. (b) + (f) $\alpha=0.95$, bad pixels: $10.7 \%$. (c) $+(\mathrm{g}) \alpha=0.5$, bad pixels: $20.9 \%$. (d) $+(\mathrm{h}) \alpha=0.1$, bad pixels: $23.5 \%$. The larger the parameter, the better the results. Hence, the gradient comparison seems more reliable than the color comparison. This is specific to this scene (lots of textures). This is not true for scenes with many flat regions (the border of the lamp here is better estimated for a smaller value of $\alpha$ ). 

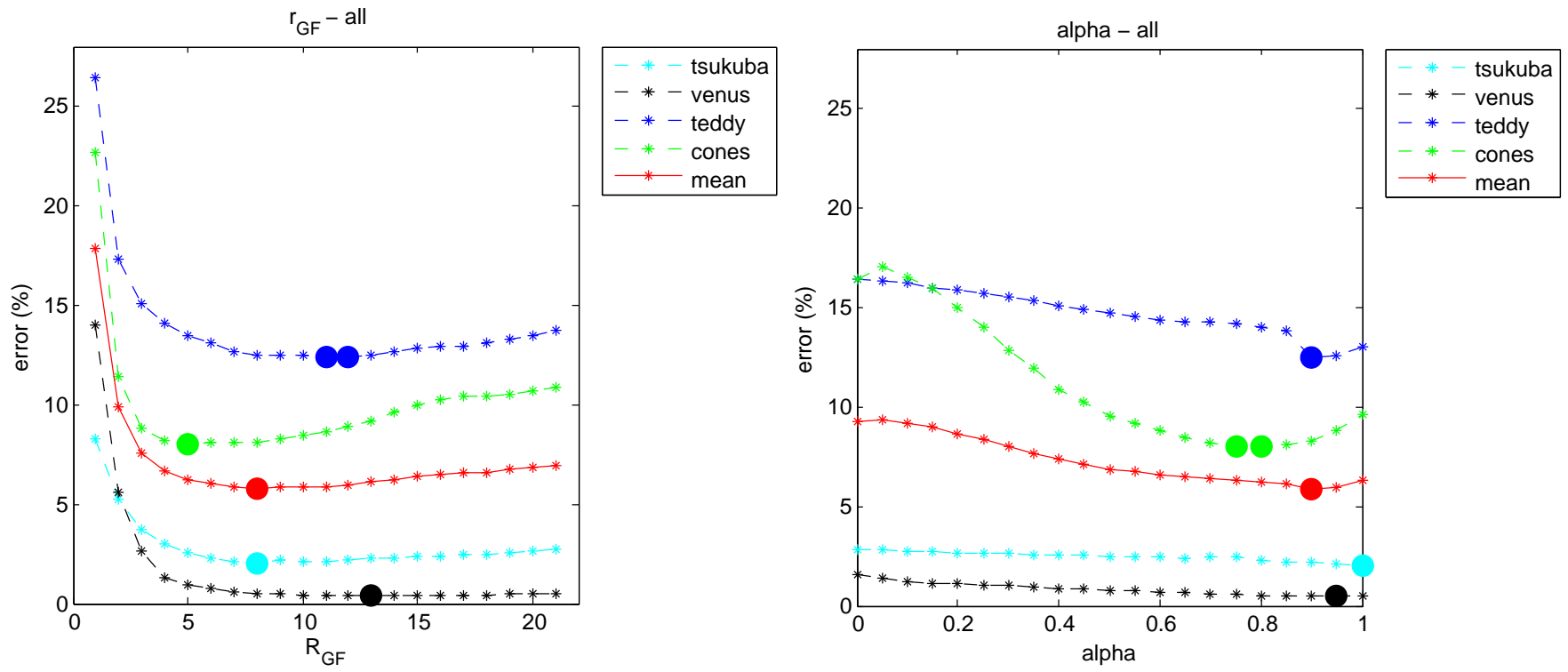

Figure 13: Influence of the parameters $r_{\mathrm{GF}}$ (left) and $\alpha$ (right) on errors in the Middlebury benchmark.

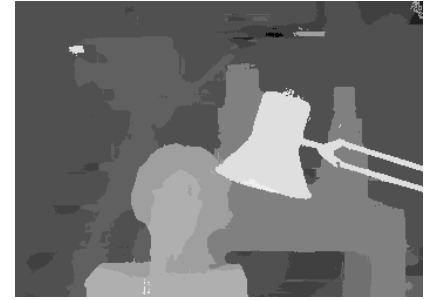

(a) $\tau_{1}=7, \tau_{2}=2$

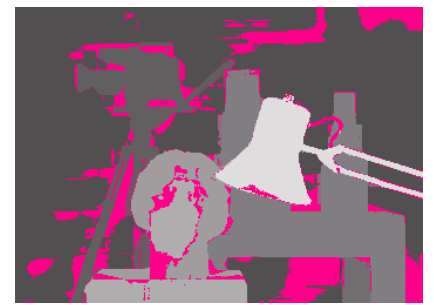

(e) $\tau_{1}=7, \tau_{2}=2$

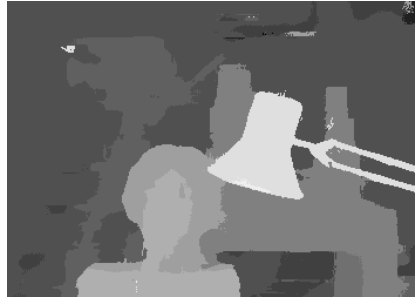

(b) $\tau_{1}=20, \tau_{2}=2$

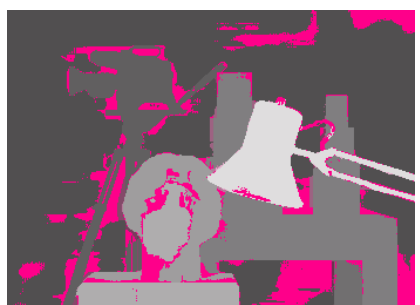

(f) $\tau_{1}=20, \tau_{2}=2$

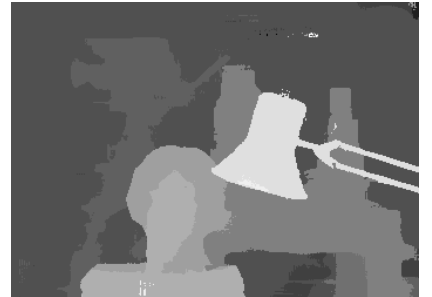

(c) $\tau_{1}=7, \tau_{2}=10$

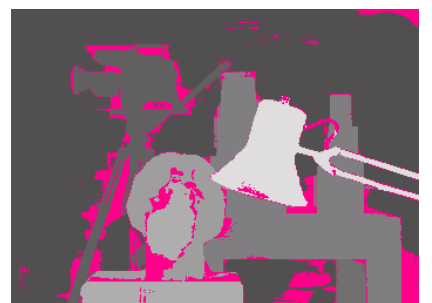

(g) $\tau_{1}=7, \tau_{2}=10$

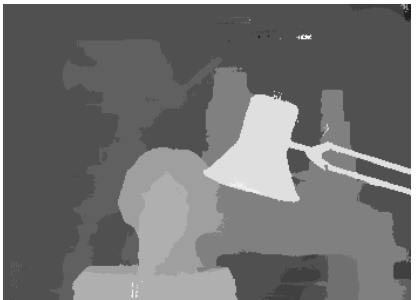

(d) $\tau_{1}=20, \tau_{2}=10$

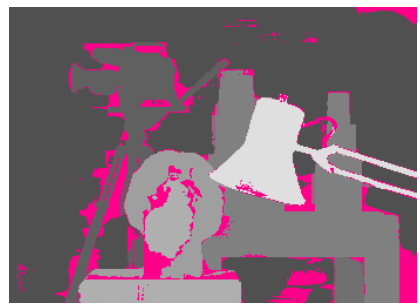

(h) $\tau_{1}=20, \tau_{2}=10$

Figure 14: Influence of thresholds $\tau_{1}$ and $\tau_{2}$. (a) $+(\mathrm{e})$ Default parameters $\left(\tau_{1}=7, \tau_{2}=2\right)$, bad pixels: 12.9\%. (b) $+(\mathrm{f}) \tau_{1}=20$, bad pixels: $12.1 \%$. (c) $+(\mathrm{g}) \tau_{2}=10$, bad pixels: $9.65 \%$. (d) $+(\mathrm{h}) \tau_{1}=20$, $\tau_{2}=10$, bad pixels: $9.49 \%$. The results improve when the thresholds are chosen a little larger. Actually, by default, they are set too small. Thus, big differences do not affect the comparison too much. However, errors appear when they are too large (typically larger than those presented here). 


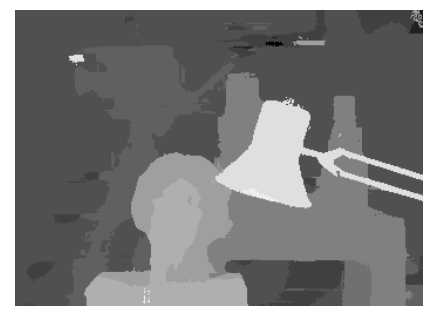

(a) $\varepsilon=255^{2} \cdot 10^{-4}$

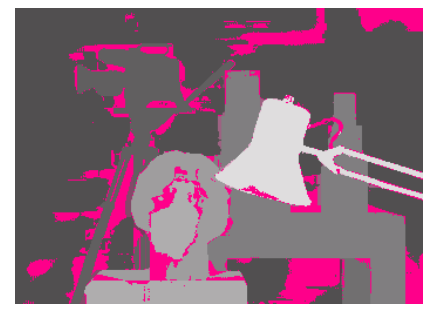

(e) $\varepsilon=255^{2} \cdot 10^{-4}$

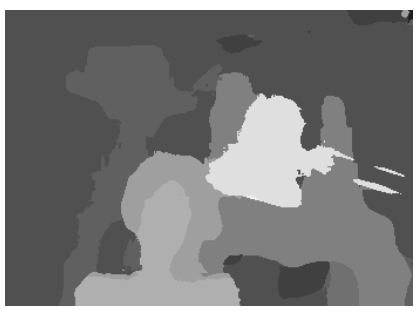

(b) $\varepsilon=255^{2} \cdot 10^{-1}$

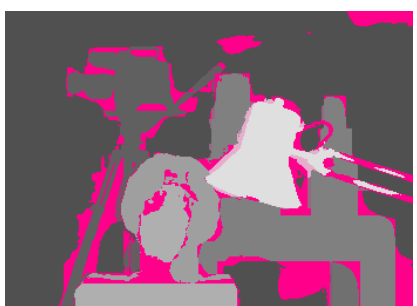

(f) $\varepsilon=255^{2} \cdot 10^{-1}$

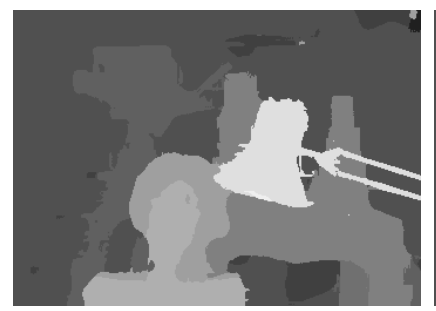

(c) $\varepsilon=255^{2} \cdot 10^{-2}$

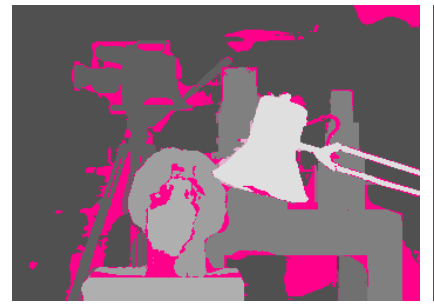

(g) $\varepsilon=255^{2} \cdot 10^{-2}$

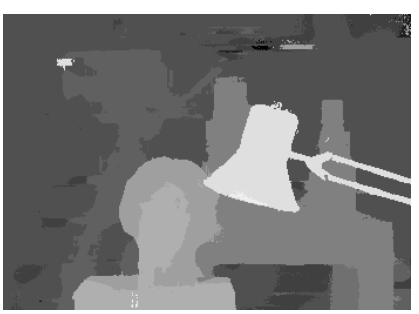

(d) $\varepsilon=255^{2} \cdot 10^{-5}$

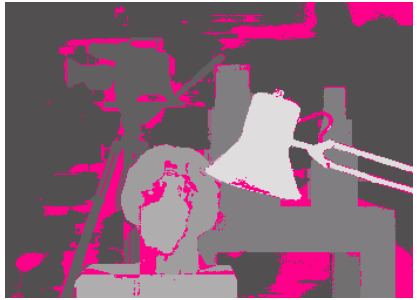

(h) $\varepsilon=255^{2} \cdot 10^{-5}$

Figure 15: Influence of the regularization parameter. (a) $+(\mathrm{e})$ Default parameters $\left(\varepsilon=255^{2} \cdot 10^{-4}\right)$. Bad pixels: $12.9 \%$. (b) + (f) $\varepsilon=255^{2} \cdot 10^{-1}$, bad pixels: $13.5 \%$. (c) + (g) $\varepsilon=255^{2} \cdot 10^{-2}$, bad pixels: $12.2 \%$. (d) $+(\mathrm{h}) \varepsilon=255^{2} \cdot 10^{-5}$, bad pixels: $13.0 \%$. For large values of $\varepsilon$, the object boundaries are loose and the disparity map is smoother.

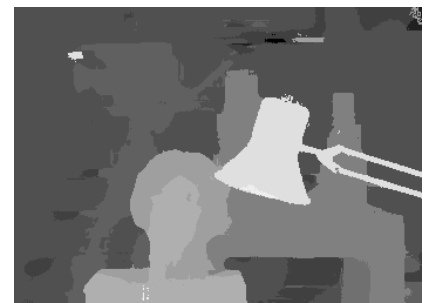

(a) $r_{\mathrm{WMF}}=9$

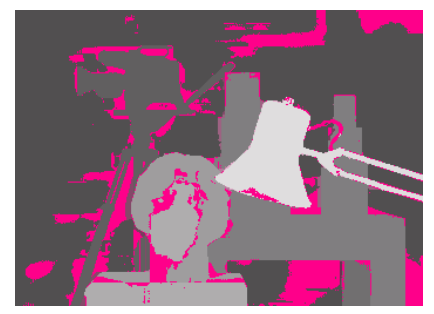

(e) $r_{\mathrm{WMF}}=9$

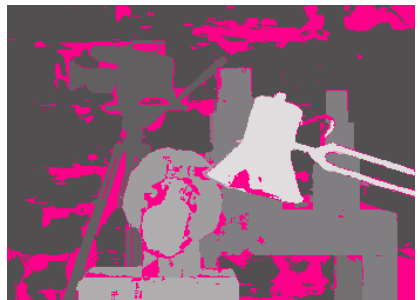

(b) $r_{\mathrm{WMF}}=4$

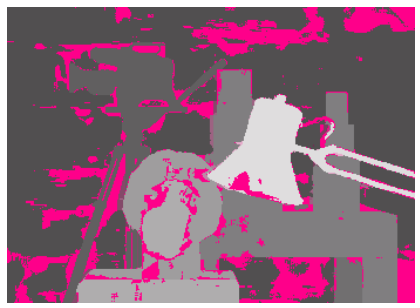

(f) $r_{\mathrm{WMF}}=4$

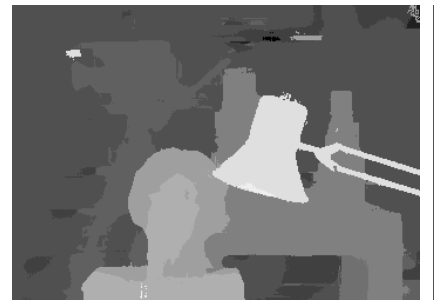

(c) $r_{\mathrm{WMF}}=19$

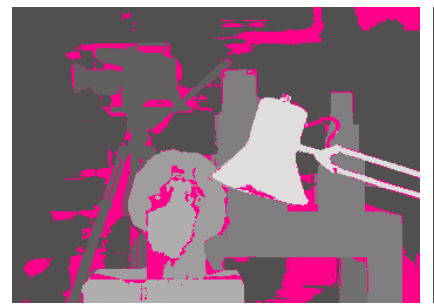

(g) $r_{\mathrm{WMF}}=19$

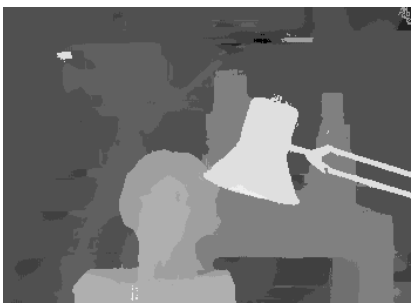

(d) $r_{\mathrm{WMF}}=29$

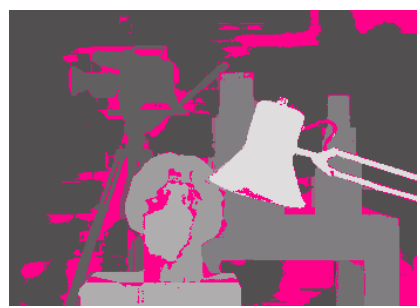

(h) $r_{\mathrm{WMF}}=29$

Figure 16: Influence of the size of the weighted median filter. (a) $+(\mathrm{e})$ Default parameters $\left(r_{\mathrm{WmF}}=9\right)$, bad pixels: $13.0 \%$. (b) + (f) $r_{\mathrm{WMF}}=4$, bad pixels: $13.2 \%$. (c) + (g) $r_{\mathrm{WMF}}=19$, bad pixels: $12.9 \%$. $(\mathrm{d})+(\mathrm{h}) r_{\mathrm{WMF}}=29$, bad pixels: $12.8 \%$. This parameter shows no influence on the results. However, as expected, the results are slightly better when the filter size grows. 


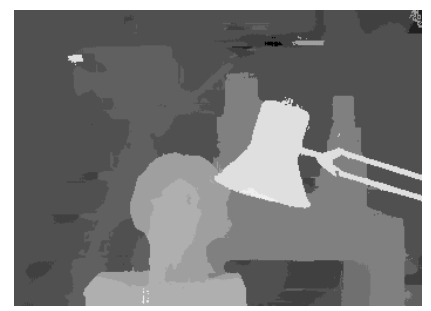

(a) $\sigma_{\mathrm{c}}=255 \cdot 10^{-1}$

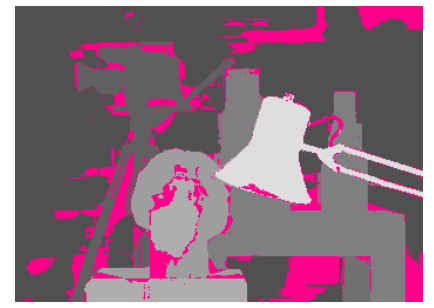

(e) $\sigma_{\mathrm{c}}=255 \cdot 10^{-1}$

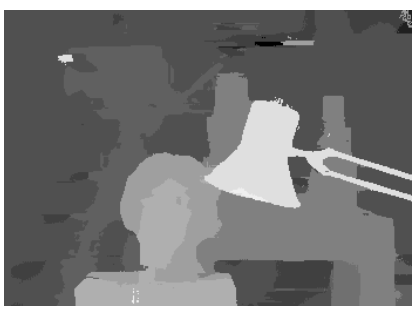

(b) $\sigma_{\mathrm{c}}=255 \cdot 10^{-2}$

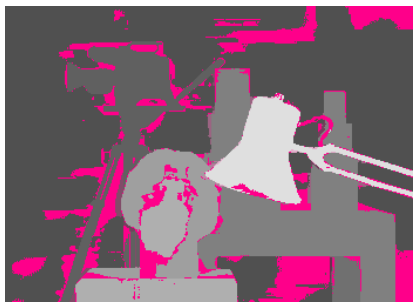

(f) $\sigma_{\mathrm{c}}=255 \cdot 10^{-2}$

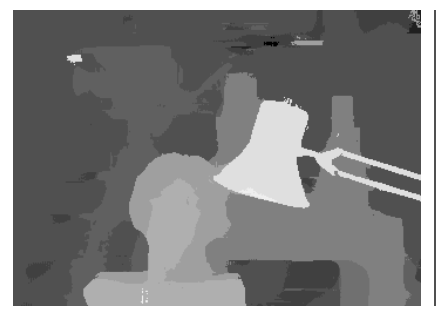

(c) $\sigma_{\mathrm{c}}=255$

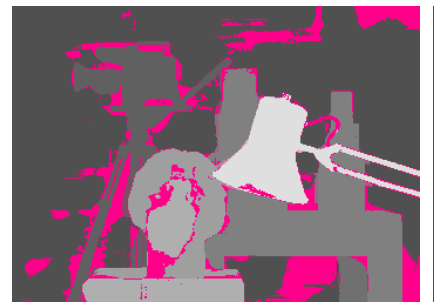

(g) $\sigma_{\mathrm{c}}=255$

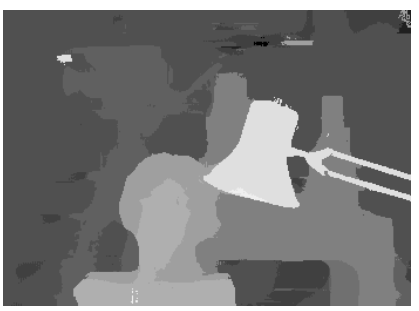

(d) $\sigma_{\mathrm{c}}=255 \times 4$

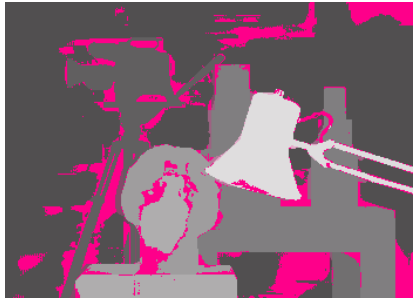

(h) $\sigma_{\mathrm{c}}=255 \times 4$

Figure 17: Influence of the parameter $\sigma_{\mathrm{c}} \cdot(\mathrm{a})+(\mathrm{e})$ Default parameters $\left(\sigma_{\mathrm{c}}=255 \cdot 10^{-1}\right)$, bad pixels: $12.9 \%$. (b) + (f) $\sigma_{\mathrm{c}}=255 \cdot 10^{-2}$, bad pixels: $13.3 \%$. (c) $+(\mathrm{g}) \sigma_{\mathrm{c}}=255$, bad pixels: $12.7 \%$. (d) $+(\mathrm{h})$ $\sigma_{\mathrm{c}}=255 \times 4$, bad pixels: $12.8 \%$. This parameter has no strong influence, but it should not be chosen too large or too small.

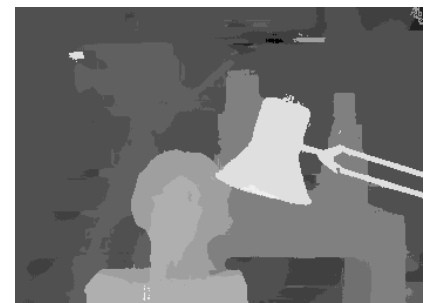

(a) $\sigma_{\mathrm{s}}=9$

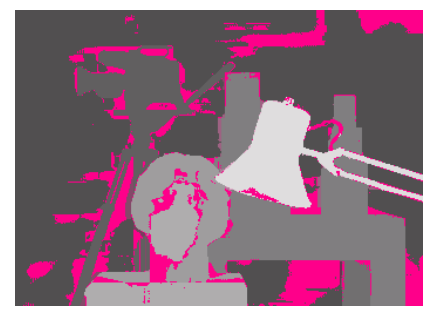

(e) $\sigma_{\mathrm{s}}=9$

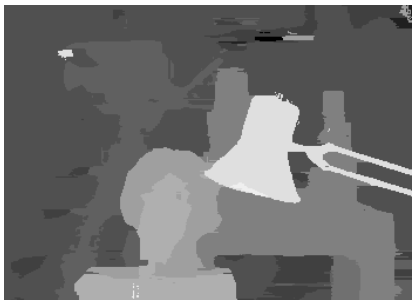

(b) $\sigma_{\mathrm{s}}=10^{-1}$

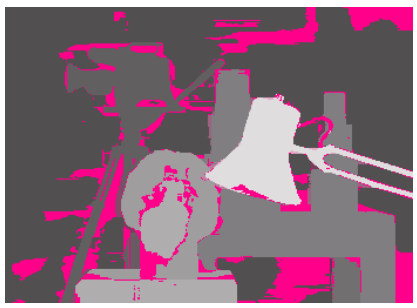

(f) $\sigma_{\mathrm{s}}=10^{-1}$

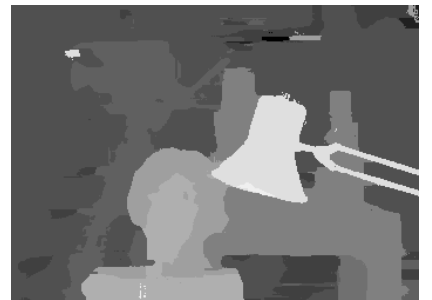

(c) $\sigma_{\mathrm{s}}=2$

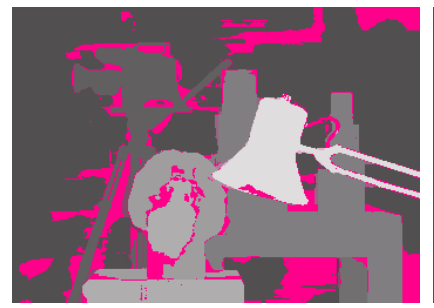

(g) $\sigma_{\mathrm{s}}=2$

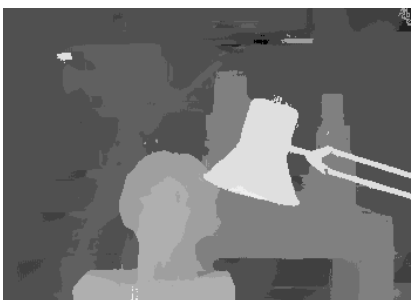

(d) $\sigma_{\mathrm{s}}=15$

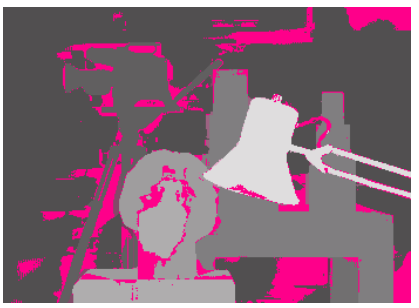

(h) $\sigma_{\mathrm{s}}=15$

Figure 18: Influence of the parameter $\sigma_{\mathrm{s}}$. (a) $+(\mathrm{e})$ Default parameters $\left(\sigma_{\mathrm{s}}=9\right)$, bad pixels: $12.9 \%$. (b) + (f) $\sigma_{\mathrm{s}}=10^{-1}$, bad pixels: $13.4 \%$. (c) $+(\mathrm{g}) \sigma_{\mathrm{s}}=2$, bad pixels: $13.3 \%$. (d) $+(\mathrm{h}) \sigma_{\mathrm{s}}=15$, bad pixels: $12.7 \%$. This parameter does not change the results a lot, but it should not be chosen too large or too small. 
Then, the parameters $\sigma_{\mathrm{c}}$ (Figure 17) and $\sigma_{\mathrm{s}}$ (Figure 18) are tuned, which control the importance of the color distance and the spatial distance in the bilateral filter. The experiments do not uncover any significant changes. One can notice that when these parameters are too large, the results are worse, because the bilateral filter is then not discriminatory enough. On the contrary, when they are too small, the filter rejects too many pixels.

By tuning properly the parameters to get better results, we managed to reach $9.01 \%$ bad pixels (and $8.44 \%$ in nonoccluded regions). Figure 19 shows the disparity map obtained with the following parameter values: $r_{\mathrm{GF}}=11, \alpha=0.95, \tau_{1}=20, \tau_{2}=10, \varepsilon=255^{2} \cdot 10^{-4}, r_{\mathrm{WMF}}=29, \sigma_{\mathrm{c}}=255 \cdot 10^{-1}$, $\sigma_{\mathrm{s}}=15$. However, one should keep in mind that the optimal values of the parameters depend on the scene.

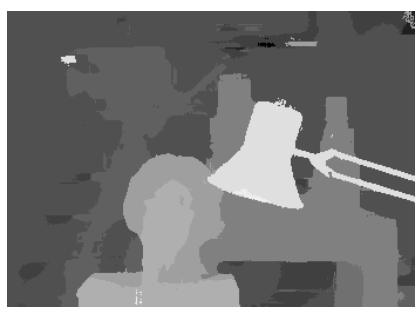

(a) Default parameters

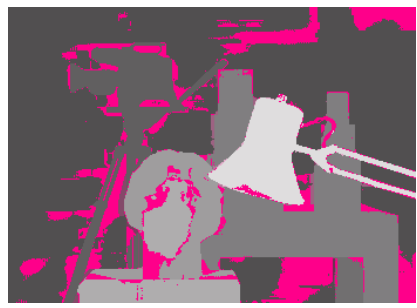

(c) Default parameters

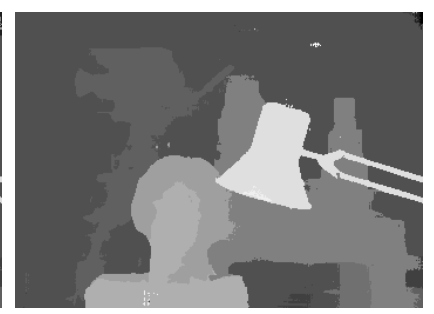

(b) Tuned parameters

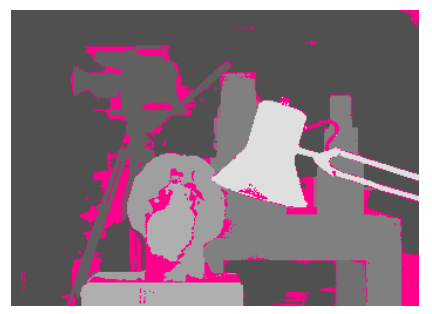

(d) Tuned parameters

Figure 19: Enhancement by tuning properly the parameters. (a)+(c) Default parameters. Bad pixels: 12.9\%. (b) + (d) Tuned parameters. Bad pixels: 9.01\%. We managed to enhance the result, especially in the background of the scene, while keeping a good estimation anywhere else. Nevertheless, one can notice that the disparity map is smoother, which creates some errors (e.g. on the right border of the lamp or on the statue).

We can compare the result before and after the densification process. Figure 20 shows the disparity map obtained with and without this step. The densification cleans the disparity map, by removing big errors (e.g. on the lamp). However, some details are lost (e.g. the wire on the lamp). Actually, one can notice that the densification step mainly enhances the result on flat regions of the disparity map. It does not affect much discontinuity regions. Then, one should consider this step to be a filtering step, as it removes big errors in flat regions.

\section{$5 \quad$ Examples}

\subsection{Comparison with Original Algorithm}

The authors of the original algorithm [8] propose a Matlab implementation ${ }^{6}$. It performs similarly to our implementation, the slight differences being attributed to:

- Image color to gray conversion formulas.

\footnotetext{
${ }^{6}$ https://www.ims.tuwien.ac.at/publications/tuw-202088
} 


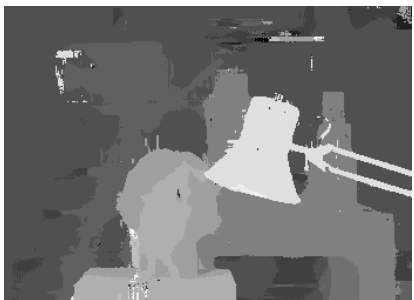

(a) Without densification

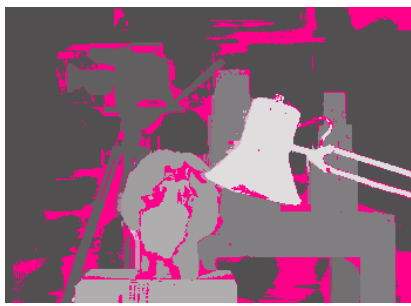

(c) Without densification

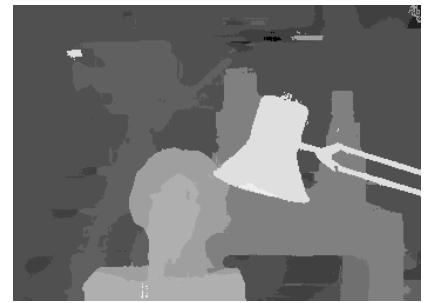

(b) With densification

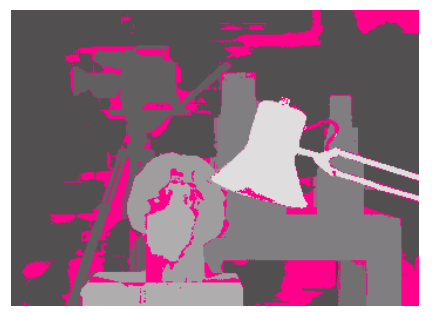

(d) With densification

Figure 20: Enhancement by the densification process. (a)+(c) Without densification. Bad pixels: 14.5\%. (b)+(d) With densification. Bad pixels: 12.9\%. The densification mainly enhanced the results on nonoccluded regions. It removed some obvious errors easily detected (e.g. on the lamp or on the statue), but it also removed some details (e.g. the wire on the lamp).

Error threshold $=1$ :

\begin{tabular}{c|c|c|ccc|ccc|ccc|ccc}
\hline Impl. & AR & Rank & \multicolumn{3}{|c|}{ Tsukuba } & \multicolumn{3}{|c|}{ Venus } & \multicolumn{3}{c}{ Teddy } & \multicolumn{3}{c}{ Cones } \\
\hline Ours & 50.5 & 49 & 1.92 & 2.24 & 7.68 & 0.26 & 0.47 & 2.55 & 6.98 & 12.4 & 16.7 & 2.83 & 8.25 & 7.99 \\
Authors & 38.0 & 27 & 1.51 & 1.85 & 7.61 & 0.20 & 0.39 & 2.42 & 6.16 & 11.8 & 16.0 & 2.71 & 8.24 & 7.66
\end{tabular}

Error threshold $=0.5$ :

\begin{tabular}{c|c|c|ccc|ccc|ccc|ccc}
\hline Impl. & AR & Rank & \multicolumn{3}{|c|}{ Tsukuba } & \multicolumn{3}{|c|}{ Venus } & \multicolumn{3}{|c}{ Teddy } & \multicolumn{3}{c}{ Cones } \\
\hline Ours & 38.1 & 28 & 11.5 & 11.9 & 16.1 & 5.74 & 6.17 & 10.4 & 12.1 & 18.5 & 26.0 & 8.16 & 13.9 & 15.6 \\
Authors & 35.9 & 26 & 11.2 & 11.7 & 15.6 & 5.99 & 6.43 & 10.8 & 11.3 & 18.1 & 25.3 & 7.71 & 13.7 & 15.1
\end{tabular}

Table 2: Results on Middlebury stereo benchmark ( $\mathrm{AR}=$ Average Rank). First implementation is our code, the second one is the authors' CUDA code results, as stored in the benchmark.

- Handling of cost for pixels outside the image, term $N(i, i+d)$ of $(8)$ : the Matlab code assumes a constant color $R=G=B=b$ with $b=3$ of $I_{R}$ outside its boundaries, while we assign the maximum possible cost.

- Numerical inaccuracies.

A comparison on the Middlebury stereo benchmark is given in Table 2. The authors' implementation is not their Matlab code. The latter uses $d_{\max }=-1$ instead of 0 , but otherwise gives results similar to our implementation. The CUDA code (not available) gives sensibly better results for the error threshold $=1$ (only disparity errors strictly larger than 1 pixel are reported). Comparing both error thresholds, we see that our implementation has significantly more errors of 1 pixel.

After a personal communication with the CUDA implementation author [5], it appears that on the Tsukuba pair, the left-right consistency threshold is $d_{L R}=1$ instead of 0 . Whereas taking such a threshold yields slightly better results on Tsukuba and Cones, it deteriorates Venus and Teddy, so does not improve the final rank in the benchmark.

Moreover, we see in the green areas of Figure 21 some wrong disparity pixels that are not detected as occluded with the CUDA code but are still corrected in the final map. This is inconsistent with 

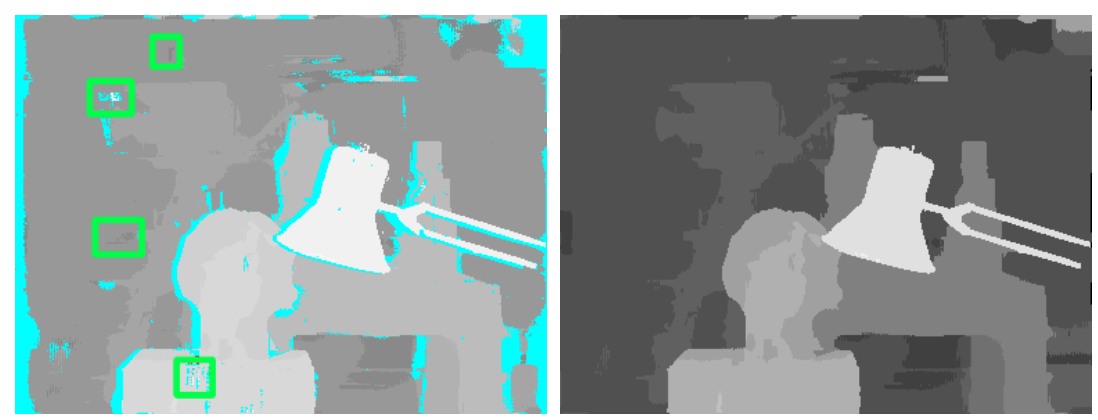

Figure 21: CUDA implementation result on Tsukuba pair. Left: disparity map with occluded pixels. Right: final disparity map.

the described algorithm. No explanation could be found.

\subsection{Results}

We now show some other results obtained by our implementation. The experiments were done on images without ground truth. Hence, the results can only be appreciated visually. If not stated otherwise, the results shown were obtained by the whole process (including occlusion detection, filling and post-processing). The algorithm was launched with the default parameters (see previous section).

Globally, the results tend to show that this method gives very good results. The default parameters are fairly well chosen, which is sufficient most of the time. Hence, one can launch the code without tuning any parameter and yet get satisfactory results. Nevertheless, they can be enhanced by tuning the parameters, which are numerous. Thus, one has to tune and test each parameter several times to find the optimal parameters.

Chair. The code was tested on the Chair pair (Figure 22). Results are fairly good. However note that this pair is easy to deal with, since almost none of the stereovision difficulties are encountered (almost no reflection, textured regions).

This result is compared with the result obtained before the left-right consistency check (Figure 23). Results are visually better. However, Figure 24 shows that this process degrades some parts of the disparity map, especially on some objects boundaries. Densification leads to better overall results, but some accuracy is lost.

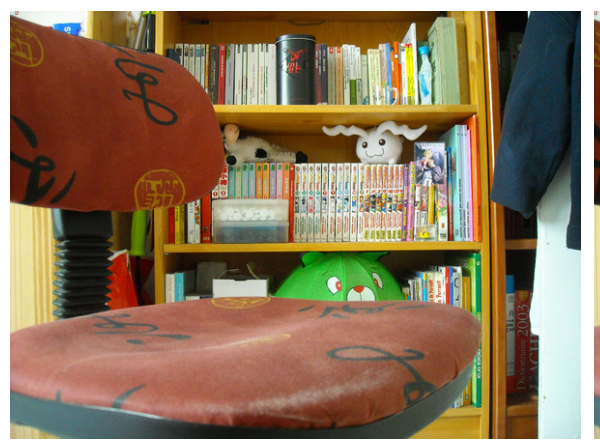

(a)

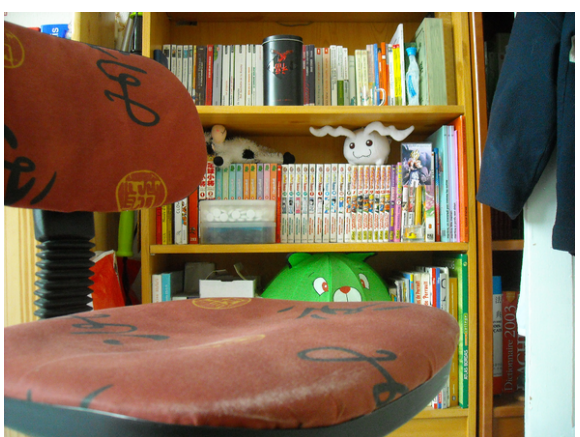

(b)

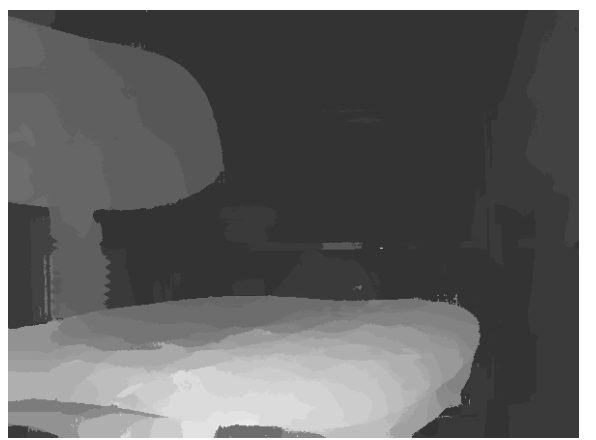

(c)

Figure 22: Chair pair. (a) Left image. (b) Right image. (c) Result. The result is very satisfactory, the depth of the scene seems probable. 
Umbrella. Results on the Umbrella pair are shown in Figure 25. This experiment discloses a strong fattening effect, that is, neighbor points of objects in the foreground are labelled as if they belonged to the objects. Hence, such objects appear fatter than they actually are. This specific example shows that the boundaries of the green umbrella are not clearly drawn, since they are badly estimated (e.g. the left ear is bigger than expected). The background adopted the umbrella disparity.

\section{Discussion on Adaptive-Window Approaches}

The effectiveness of the method described in this article mainly relies on the use of the guided filter. Formula (17) expresses this filter with adaptive weights, which are shown in Figure 26. Hence, the filter only takes into account some selected neighboring pixels. The window is thus adapted to each pixel.

To compute the weights associated to a pixel $i$, we can use (17) or (23), but another possibility is for each pixel $j$ to apply the guided filter to the Dirac image $p_{j}(k)=\delta_{j k}$ (1 at pixel $j$, 0 everywhere else) and take the filtered image at $i, W_{i j}^{\mathrm{GF}}=q_{j}(i)$. Note that only pixels $j$ at $l^{\infty}$-distance at most $2 \cdot r_{\mathrm{GF}}$ from $i$ should be considered, the sum in (23) reducing to 0 otherwise.

Similar strategies, based on adaptive windows, have been proposed previously. Indeed, local stereo-matching methods highly rely on window comparison. Such comparison assumes that the depth within the window does not vary much, which is not the case on depth discontinuities. Hence, local methods require choosing the best window to perform well. The choice of a window depends on many criteria. Windows have to be large enough to avoid low signal to noise ratio. However, when they are too large, they may cover regions in which the depth varies a lot. Indeed, in such regions, the comparison may be affected by projective distortion due to strong depth discontinuities. Accordingly, several approaches have been tested to design adaptive windows. This strategy is aimed at selecting for each pixel the appropriate window.

One of the former approaches consists in adapting the window size to the pixel, knowing that it should depend on local variations of intensity and disparity [6]. Indeed, high variations of disparity mean depth discontinuities, which often entails distortions. Unfortunately, this method needs to evaluate the variation of the disparity, so it strongly depends on the initial disparity estimation.

Another approach is to adapt the shape of the window to fit with the shape of the discontinuities. Boykov et al. [1] find for each pixel an arbitrarily shaped connected window chosen by evaluating the plausibility of each pixel to have the same disparity as the pixel under consideration. Veksler $[11,12]$

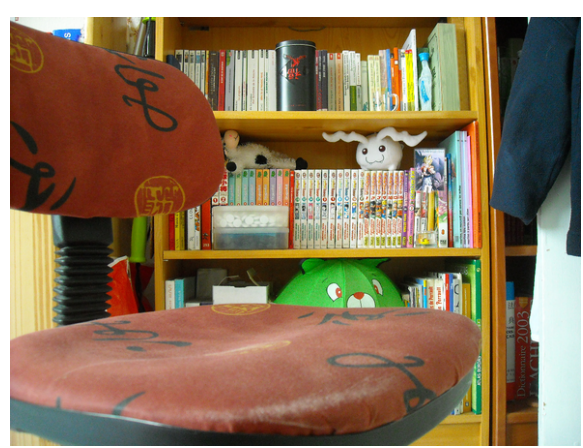

(a)

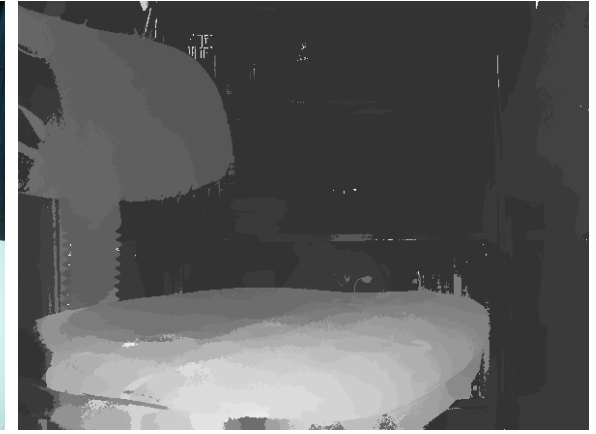

(b)

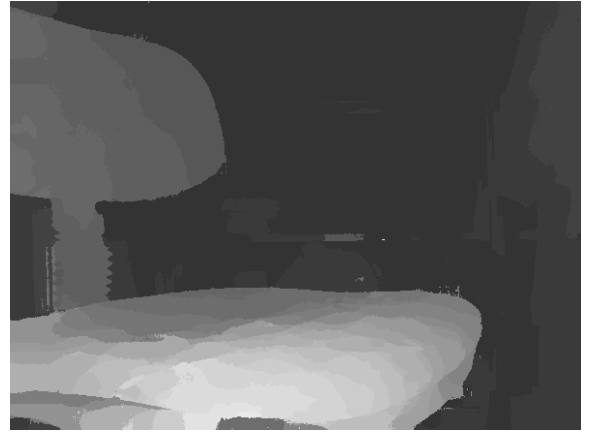

(c)

Figure 23: Chair pair. (a) Left image. (b) Before the occlusion detection. (c) After the whole process. After the occlusion detection and the densification step, the disparity map is cleaner, most of obvious errors have been removed. Moreover, the disparity has been well interpolated on the left border of the image. 


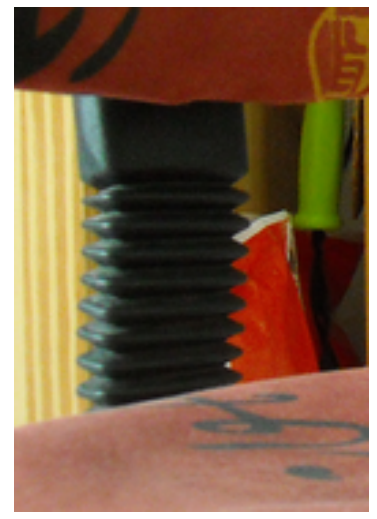

(a)

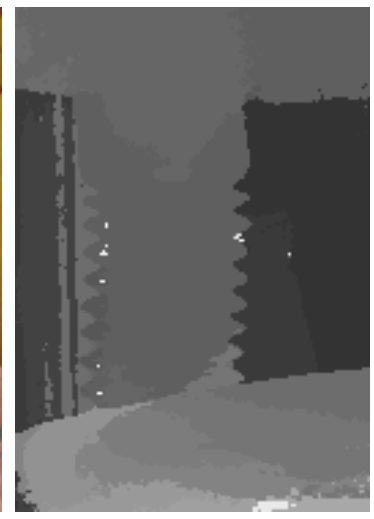

(b)

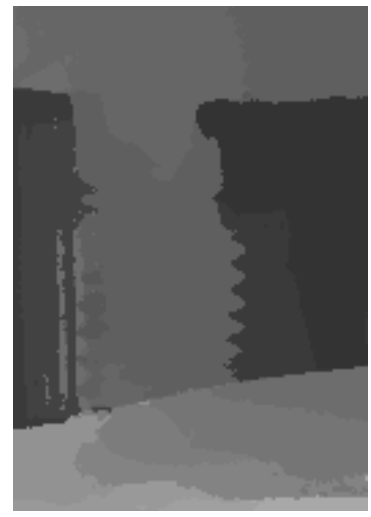

(c)

Figure 24: Chair pair (detail). (a) Left image. (b) Before the occlusion detection. (c) After the whole process. In this detail, the disparity was better estimated before the occlusion detection, even though the densification process removed some errors.

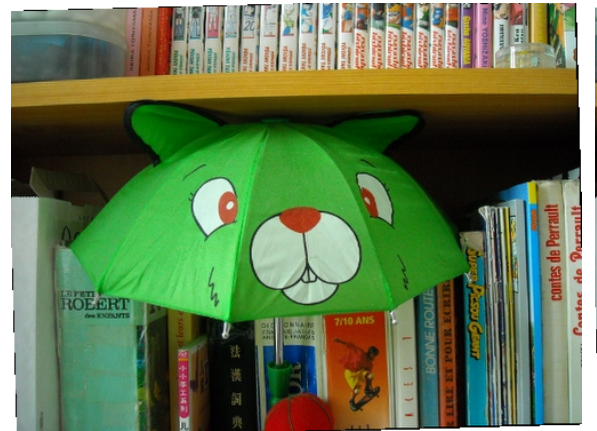

(a)

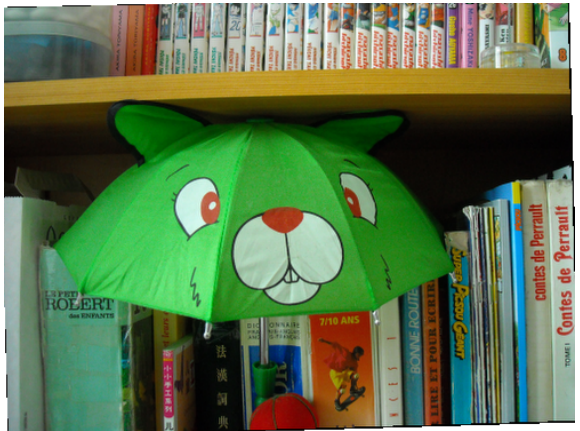

(b)

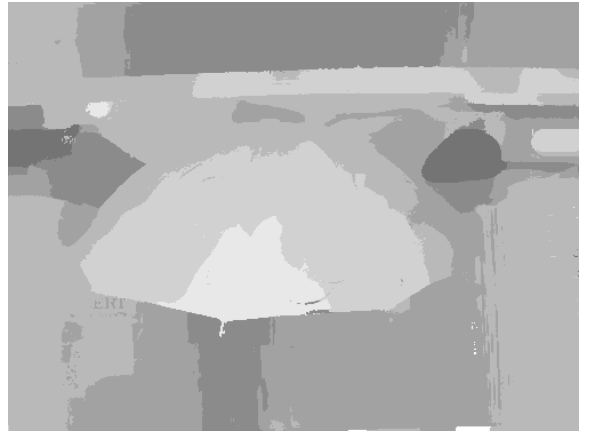

(c)

Figure 25: Umbrella pair. (a) Left image. (b) Right image. (c) Result.

uses a set of windows (called compact windows) of different size and shape, and chooses the most reliable (with minimal average error). However, this method does not consider general shapes of window, which limits its performance.

More recently, a new adaptive-window method, proposed by [14], uses square weighted windows. For each pixel, the weights are computed so that only neighboring pixels of interest (i.e. belonging to the same object) have heavy weight. Since the Gestalt principles state that grouping rules based on similarity and proximity are among the strongest, the weights should depend on the color similarity and the spatial proximity. The weights are chosen so that they decrease quickly while the pixels $i$ and $j$ are dissimilar or not close enough:

$$
W_{i j}:=\frac{1}{K_{i}} \exp \left(-\frac{\|i-j\|}{\sigma_{\mathrm{s}}^{2}}\right) \cdot \exp \left(-\frac{\left\|I_{L}(i)-I_{L}(j)\right\|_{c}}{\sigma_{\mathrm{c}}^{2}}\right)
$$

where $\|\cdot\|_{c}$ denotes the Euclidean distance between two colors represented in color space ${ }^{7}$. Note that this is close to the bilateral filter used in the post-processing. Figure 26 shows examples of weights obtained by this formula. A limitation of this method is that it is computationally expensive, while the guided filter discloses similar results with a lower complexity. Note that the name "bilateral

\footnotetext{
${ }^{7}$ The original article [14] uses Euclidean distance in CIELab color space, in our experiments we use $L^{1}$ distance in RGB space.
} 


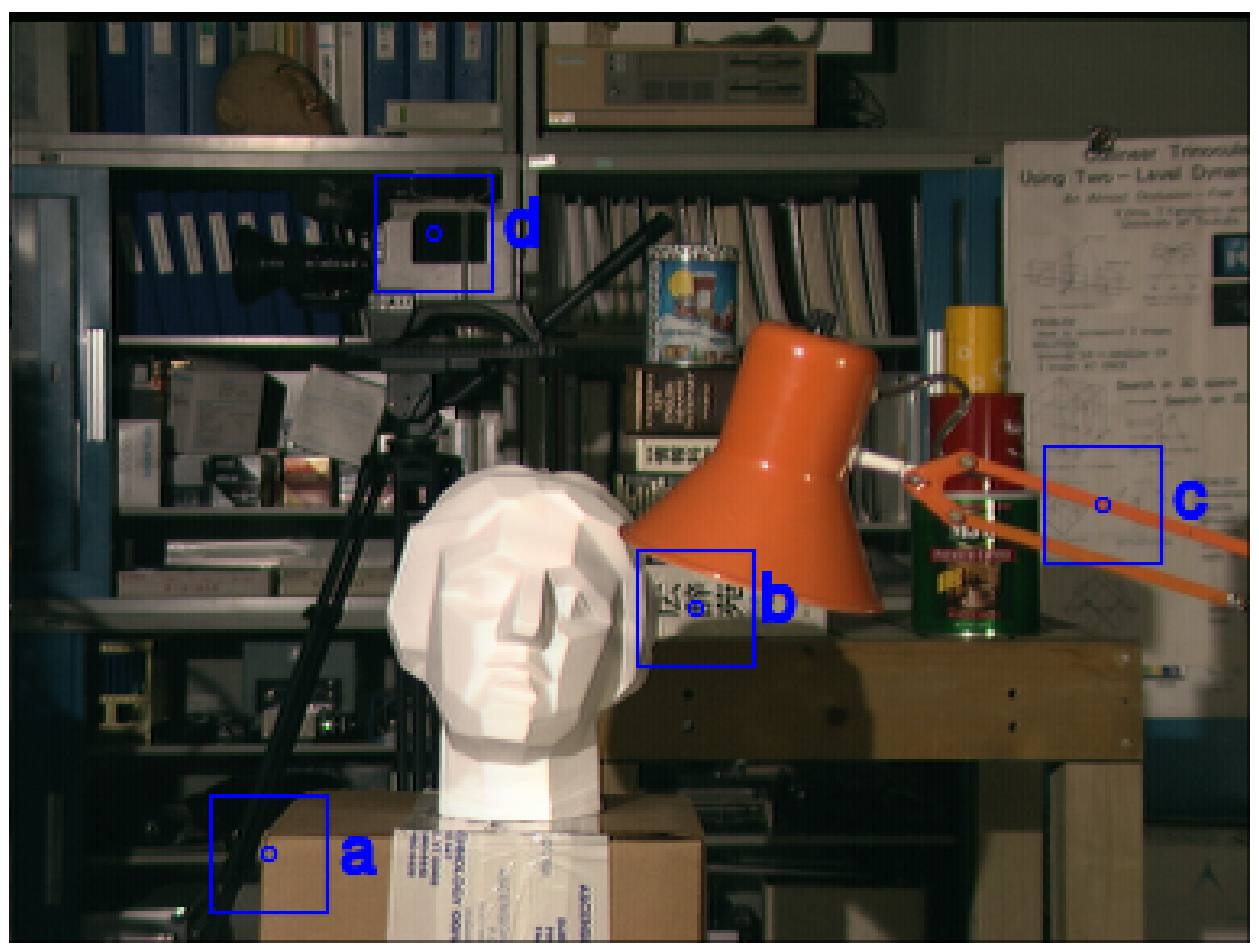

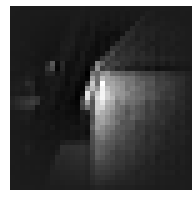

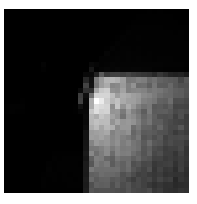

(a)

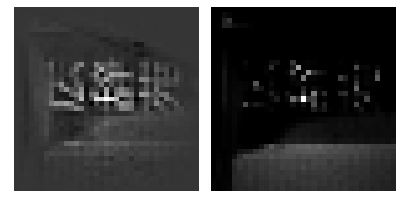

(b)

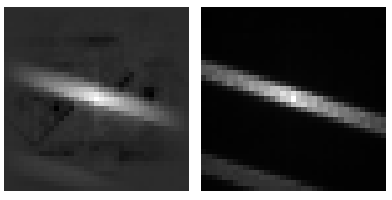

(c)

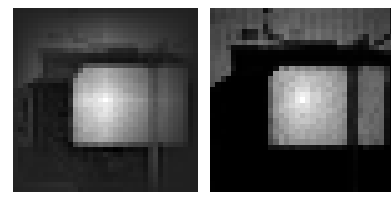

(d)

Figure 26: Adaptive weights in the guided filter (left image for each patch) and of Yoon-Kweon's bilateral filter [14] (right image for each patch).

filter" [10] is the most recent one for a filter known as the sigma filter [7], the Yaroslavsky filter [13] and the SUSAN filter [9].

The bilateral weights in Figure 26 are computed with parameters: $\sigma_{\mathrm{s}}^{2}=17.5$ (half the window size $35 \times 35)$ and $\sigma_{\mathrm{c}}^{2}=14 \cdot 3$ (3 channels). The weights appear roughly equivalent by both methods. Note that there is implicitly a decreasing tendency with the distance to central pixel $i$ in the guided filter, because the further pixel $j$, the fewer windows $\omega_{k}$ containing $i$ and $j$. A full comparison with other adaptive-window methods will be the object of future work.

\section{Image Credits}

All images by the authors (license CC-BY-SA) except:

Middlebury.

\section{References}

[1] Y. Boykov, O. VeKsler, And R. ZABIH, A variable window approach to early vision, IEEE Transactions on Pattern Analysis and Machine Intelligence (PAMI), 20 (1998), pp. 1283-1294. http://dx.doi.org/10.1109/34.735802. 
[2] F.C. Crow, Summed-area tables for texture mapping, ACM SIGGRAPH Computer Graphics, 18 (1984), pp. 207-212. http://dx.doi.org/10.1145/964965.808600.

[3] G. Facciolo, N. Limare, and E. Meinhardt-Llopis, Integral images for block matching. preprint, http://www.ipol.im/pub/pre/57, 2013.

[4] K. He, J. Sun, And X. TAng, Guided image filtering, in 11th European Conference on Computer Vision, Springer, 2010, pp. 1-14. http://dx.doi.org/10.1007/978-3-642-15549-9_1.

[5] A. Hosni. Personal communication (Feb. 2013).

[6] T. Kanade And M. OKutomi, A stereo matching algorithm with an adaptive window: theory and experiment, IEEE Transactions on Pattern Analysis and Machine Intelligence (PAMI), 16 (1994), pp. 920-932. http://dx.doi.org/10.1109/34.310690.

[7] J-S. LEE, Digital image smoothing and the sigma filter, Computer Vision, Graphics, and Image Processing, 24 (1983), pp. 255-269. http://dx.doi.org/10.1016/0734-189X (83)90047-6.

[8] C. Rhemann, A. Hosni, M. Bleyer, C. Rother, and M. Gelautz, Fast cost-volume filtering for visual correspondence and beyond, in IEEE Conference on Computer Vision and Pattern Recognition (CVPR), IEEE, 2011, pp. 3017-3024. http://dx . doi .org/10.1109/CVPR. 2011.5995372.

[9] S.M. Smith And J.M. Brady, Susan-a new approach to low level image processing, International Journal of Computer Vision (IJCV), 23 (1997), pp. 45-78. http://dx.doi.org/10. 1023/A : 1007963824710 .

[10] C. Tomasi and R. Manduchi, Bilateral filtering for gray and color images, in 6th International Conference on Computer Vision (ICCV), IEEE, 1998, pp. 839-846. http://dx.doi.org/ 10.1109/ICCV.1998.710815.

[11] O. VEKSLER, Stereo correspondence with compact windows via minimum ratio cycle, IEEE Transactions on Pattern Analysis and Machine Intelligence (PAMI), 24 (2002), pp. 1654-1660. http://dx.doi.org/10.1109/TPAMI.2002.1114859.

[12] _ Fast variable window for stereo correspondence using integral images, in IEEE Computer Society Conference on Computer Vision and Pattern Recognition (CVPR), vol. 1, IEEE, 2003, pp. I-556. http://dx.doi.org/10.1109/CVPR.2003.1211403.

[13] L.P. Yaroslavsky, Digital picture processing: an introduction., Springer-Verlag New York, Inc., ISBN 3-540-11934-5, 1985.

[14] K-J. YoOn AND I-S. KWEOn, Adaptive support-weight approach for correspondence search, IEEE Transactions on Pattern Analysis and Machine Intelligence (PAMI), 28 (2006), pp. 650656. http://dx.doi.org/10.1109/cvpr.2005.218. 